\title{
Longitudinal acoustic instabilities in slender solid propellant rockets: linear analysis
}

\author{
By J. E. GARCÍA-SCHÄFER AND A. LIÑÁN \\ Escuela de Ingenieros Aeronáuticos, Universidad Politécnica de Madrid, \\ Plaza del Cardenal Cisneros 3, 28040 Madrid, Spain
}

(Received 12 June 1998 and in revised form 19 October 2000)

To describe the acoustic instabilities in the combustion chambers of laterally burning solid propellant rockets the interaction of the mean flow with the acoustic waves is analysed, using multiple scale techniques, for realistic cases in which the combustion chamber is slender and the nozzle area is small compared with the cross-sectional area of the chamber. Associated with the longitudinal acoustic oscillations we find vorticity and entropy waves, with a wavelength typically small compared with the radius of the chamber, penetrating deeply into the chamber. We obtain a set of differential equations to calculate the radial and axial dependence of the amplitude of these waves. The boundary conditions are provided by the acoustic admittance of the propellant surface, given by an existing analysis of the thin gas-phase reaction layer adjacent to the solid-gas interface, and of the nozzle, accounting here for the possible effect of the vorticity and entropy waves. The equations are integrated in closed form and the results provide the growth rate of the disturbances, which we use to determine the conditions for instability of the longitudinal oscillations.

\section{Introduction}

The purpose of this paper is to clarify the role of the vorticity and entropy waves that accompany acoustic oscillations in solid rocket motors in their acoustic instabilities. With this aim, we shall consider the simple case of an axis-symmetric configuration, in which the solid propellant is of cylindrical shape, bounded externally by an enclosure and with a cylindrical gaseous cavity of circular shape inside. The cavity is closed at one end by an endwall and the other end is attached to a nozzle, where the gases generated by the combustion process in the internal surface of the propellant are accelerated to supersonic speeds.

If the Mach number in the nozzle throat is close to unity, the ratio of its area, $A_{t}$, to the internal surface area, $A_{s}$, of the propellant bounding the cavity must be, as derived from mass conservation considerations, of the order of the Mach number $M_{b}=V_{b} / c_{b}$ of the gaseous combustion products leaving, with velocity $V_{b}$, the thin reaction layer adjacent to the surface of the propellant. $M_{b} \ll 1$, because $V_{b}$ is typically of the order of $1 \mathrm{~m} \mathrm{~s}^{-1}$, very small compared with the sound velocity $c_{b}$ of the burned gases. Due to the small value of $A_{t} / A_{s}$, the attenuation of any acoustic oscillations that may be excited in the nearly closed gaseous cavity is very weak, and this may contribute to the existence of combustion instabilities in the chamber. The analysis of the acoustic instabilities has received considerable attention in the literature; see, for example, the 


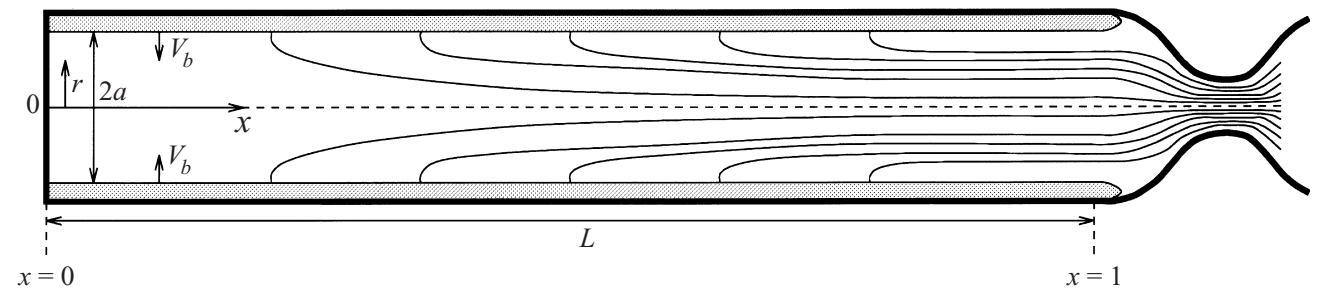

FIGURE 1. Chamber geometry, coordinate system and streamlines of the basic steady flow.

review articles of Culick \& Yang (1992), Kuentzmann (1991), T'ien (1984), and the special issue (July-August 1995) of the Journal of Propulsion and Power, notably the paper of Flandro (1995).

The acoustic oscillations in the chamber are strongly influenced by the quasisteady mean flow resulting from the combustion process. The chemical reactions are confined to thin layers, in the solid and gas, adjacent to the solid-gas interface. If the heat generated by the gas-phase chemical reaction must reach the solid by conduction, to contribute to its gasification, the Reynolds number $V_{b} l_{f} / v_{b}$, based on the thickness of the gas-phase reaction layer $l_{f}$ and the kinematic viscosity $v_{b}$-of the order of the thermal diffusivity $\alpha_{b}$-of the burned gases, must be of order unity at most. Because $l_{f}=\alpha_{b} / V_{b}$ is very small compared with the transverse size $a$ of the combustion chamber, the Reynolds number of the flow in the chamber $V_{b} a / v_{b}$ is very large compared with unity. Therefore the flow outside the reaction layer should be described, in a first approximation, with the Euler equations. This flow is rotational because the gas emerges from the solid, in a direction normal to the interface, with a stagnation pressure that varies along the chamber. The basic flow in the chamber is quasi-steady, because the time required to change the solid gasification rate and the chamber geometry is the flow residence time in the chamber, $a / V_{b}$, times the ratio $\rho_{s} / \rho_{b}$, of the solid and gas densities, large compared with unity.

The description of the basic flow, with streamlines sketched in figure 1, and of the acoustic instabilities is considerably simplified if we confine the analysis to slender rockets, with cylindrical combustion cavities of circular cross-section of radius $a$ small compared with its length $L$. The analysis can be simplified because the transverse component of the velocity, of order $V_{b}$, is small compared with the longitudinal component, of order $V_{b} / \epsilon^{\prime}$, where $\epsilon^{\prime}=a / L \ll 1$. Thus the gas flow, which comes out of the reaction layer with a velocity $V_{b}$ nearly normal to the solid interface, turns toward the chamber axis to become a quasi-parallel flow. The longitudinal velocity components when measured in terms of $c_{b}$ are of order $\epsilon=M_{b} / \epsilon^{\prime}$, still small compared with unity for typical rockets; with values of $\epsilon^{\prime}$ of order $1 / 20$ and $M_{b}$ of order $1 / 400, \epsilon$ is also of order $1 / 20$.

The transverse pressure variations, measured in terms of the quasi-steady value $p_{b}$ of the pressure at the centre point of the closed end of the chamber are of order $M_{b}^{2} p_{b}$, small when compared with the longitudinal pressure variations which are of order $\epsilon^{2} p_{b}$, also small compared with $p_{b}$. Thus, this pressure can be used to evaluate the steady burning rate, and therefore $V_{b}$, which can be considered as constant along the burning surface of the solid.

In slender rockets, we encounter acoustic instabilities of low frequency, associated with the longitudinal acoustic modes, with periods of order $L / c_{b}$, and high-frequency instabilities, associated with the transverse acoustic modes, with periods of order $a / c_{b}$. This paper is devoted to the linear analysis of the longitudinal acoustic instabilities 
in slender rocket combustion chambers of circular cross-section. The wavelength of these acoustic oscillations is of order $L$ and their characteristic oscillation time is $t_{a}=L / c_{b}$. The typical value of the residence time of a fluid particle in the chamber is $t_{r}=a / V_{b}$, also of the order of the ratio of $L$ to the characteristic longitudinal velocity $V_{b} / \epsilon^{\prime}$. Because $t_{a} / t_{r}=\epsilon \ll 1$, a fluid particle after entering the combustion chamber undergoes many oscillations before leaving the chamber through the nozzle. In addition to these two disparate time scales, $t_{a}$ and $t_{r}$, which will be used in the multiple scale analysis that follows, we also encounter another much longer time scale, namely the burn-out time $t_{b}=\left(a / V_{b}\right) \rho_{s} / \rho_{b}$. When describing the oscillations of a gas particle, during its residence time in the chamber, the regression of the solid surface can be ignored; however, the parameters characterizing the acoustic instabilities will change during the burning time because of the changes in $a$.

When analysing the longitudinal instabilities we encounter several regimes, depending on the value of the ratio of the expected response time of the flame, $t_{f}=l_{f} / V_{b}=\alpha_{b} / V_{b}^{2}$, to the acoustic time, $t_{a}=L / c_{b}$. The regime that we shall analyse corresponds to values of $t_{f} / t_{a}$ small compared with unity; for these values, the flame response, which is given by the existing analysis of the thin reaction layer, leads to vorticity and entropy waves in the main, non-reacting, region of the chamber. The existence of vorticity in the basic flow and in the acoustic oscillations is due, as shown by Flandro $(1974,1989,1995)$, to the no-slip condition of the gas flow coming out of the solid-gas interface. The axial velocity component is zero at this interface and grows with the distance from the solid surface, due to the effect of the longitudinal pressure gradient. Then, the gases coming out of the thin reaction layer have a steady axial velocity component, of order $\left(l_{f} / a\right) V_{b} / \epsilon^{\prime}$, negligible compared with $V_{b} / \epsilon^{\prime}$ because $l_{f} / a \ll 1$, but a non-zero value of the vorticity, determined by the requirement that the longitudinal velocity component is zero at the solid surface. Due to the oscillating character of the axial pressure gradient, a fluid particle, after coming out of the solid, gains an axial velocity that oscillates with the characteristic time $L / c_{b}$ of the acoustics. The wavelength in the radial direction of the oscillations is of the order $l_{0}=V_{b} L / c_{b}=\epsilon a \gg l_{f}$. These waves have been demonstrated experimentally and numerically; see, for example, Vuillot \& Kuentzmann (1986), Vuillot (1991), Vuillot \& Avalon (1991) and the review by Flandro (1995). There are similar radial temperature oscillations due to the heating of the fluid particles under compression by the oscillating pressure field, because they come out of the thin reaction zone with nearly constant temperature $T_{b}$-and thus with an oscillating entropy-a consequence of the small value of the characteristic response time of the reaction layer (of order $l_{f} / V_{b}$ in the gas phase) compared with $L / c_{b}$.

Although the existence of these vorticity and entropy waves is not due to viscous and heat conduction effects, these are responsible for their attenuation in a time of the order $t_{a t}=l_{0}^{2} / v_{b}$. We shall describe in this paper the attenuation of vorticity and entropy waves in the practically important distinguished limiting case $V_{b} l_{0}^{2} / v_{b} \simeq a$, when the waves, travelling with a radial velocity of order $V_{b}$, are only damped when they penetrate deeply into the chamber. This regime was also identified by Majdalani \& Van Moorhem $(1997 a, b)$ in their approximate analysis of the acoustic boundary layer in solid rocket motors. (See also the recent work of Zhao et al. (2000) for a description of the vorticity waves with velocity forcing at the endwall.) When looking at this distinguished limiting case, for which the Reynolds number $V_{b} a / v_{b}$, typically of order $a / l_{f}$, is of order $1 / \epsilon^{2}$, we also describe the cases, with smaller Reynolds numbers, when the damping occurs in a thin layer adjacent to the thinner reaction layer, or the opposite case when the damping of the waves occurs only near the axis. 
In summary, our purpose is to obtain a linear description of the longitudinal acoustic instabilities of slender solid propellant rockets, when the ratio, $\epsilon^{\prime}=a / L$, of the radius $a$ and the length $L$ of the cylindrical gaseous combustion chamber is small compared with unity, and the characteristic value $\epsilon=M_{b} / \epsilon^{\prime}$ of the Mach number of the steady axial velocity - of the order of the ratio of the nozzle throat area to the cross-sectional area of the chamber - is $\epsilon \ll 1$. In the analysis we shall take into account the existence of four different time scales: $t_{f}=l_{f} / V_{b}, t_{a}=L / c_{b}, t_{a t}=l_{0}^{2} / v_{b}$ and $t_{r}=a / V_{b}$, in addition to the much longer burning time $t_{b}$ which will not appear in our analysis involving much shorter time scales. There are also five length scales: $l_{0}=V_{b} t_{a}=\epsilon a, l_{a t}=V_{b} t_{a t}, a, L$ and the flame thickness $l_{f}=\alpha_{b} / V_{b}^{2}$ of the gas-phase reaction layer, which separates the solid propellant from the main non-reacting region of the gaseous cavity.

We shall analyse the distinguished regime when $t_{f} / t_{a} \ll 1$ and $l_{a t} / a=t_{a t} / t_{r}=$ $\epsilon^{2} V_{b} a / v_{b}$ is of order unity. In this limit the response of the thin gas-phase reaction layer is expected to be quasi-steady as described by Denison \& Baum (1961), Culick (1967), Krier et al. (1968) and T'ien (1972). The more recent work of Clavin \& Lazimi (1992) shows that, although the temperature of the combustion gases $T_{b}$ leaving the layer remains constant, the unsteady effects, coming from the solid and gas phases, modify the response function even at low frequencies. We shall give, in $\S \S 3$ and 4 , using in a consistent way a multiple scales technique, an unambiguous description of the flow and temperature field in the main non-reacting region. To obtain the required boundary conditions on the outer envelope of the domain we shall use the results of the existing analysis of the thin reaction layer and of the analysis of the unsteady non-uniform flow in the nozzle, given in $\S 5$. The linear stability exponent of the system is obtained in $\S 6$. The discussion of the results for the stability domain is presented in $\S 7$, where we use the solid propellant response function calculated by Clavin \& Lazimi (1992) to show the influence of the main parameters of the propellant and the chamber.

We shall not consider in this paper the other distinguished regime in which $t_{f} \sim t_{a} \sim t_{a t}$, and as a consequence $l_{f} \sim l_{0} \sim l_{a t}$, when, as described by Clavin \& Lazimi (1992), unsteady effects play a significant role in the reaction layer and the vorticity and entropy waves are attenuated before the fluid particles leave the layer.

\section{Quasi-steady flow field}

The rotational flow field of the combustion gases after leaving the thin reaction layer was described by Culick (1966) for cylindrical axisymmetric chambers, neglecting the density variations in the chamber under the assumption that the Mach number is small. An experimental confirmation of this flow description was given by Dunlap, Willoughby \& Hermsen (1974), using a cylindrical chamber with porous wall. The structure of the rotational flow of incompressible fluids in slender chambers with porous walls had been described previously by Taylor (1956). The effects of compressibility, which are only important when the Mach number is not small compared with unity, were included for the flow in slender chambers by Balakrishnan, Liñán \& Williams (1992).

The incompressible flow field described by Culick (1966) corresponds to a selfsimilar solution of the Euler equations, with the stream function, $\Psi$, and the axial and radial velocity components, $U$ and $V$, given by

$$
\Psi / \epsilon a^{2} c_{b}=\tilde{\Psi}=x \sin \left(\frac{1}{2} \pi r^{2}\right)=x F(r),
$$




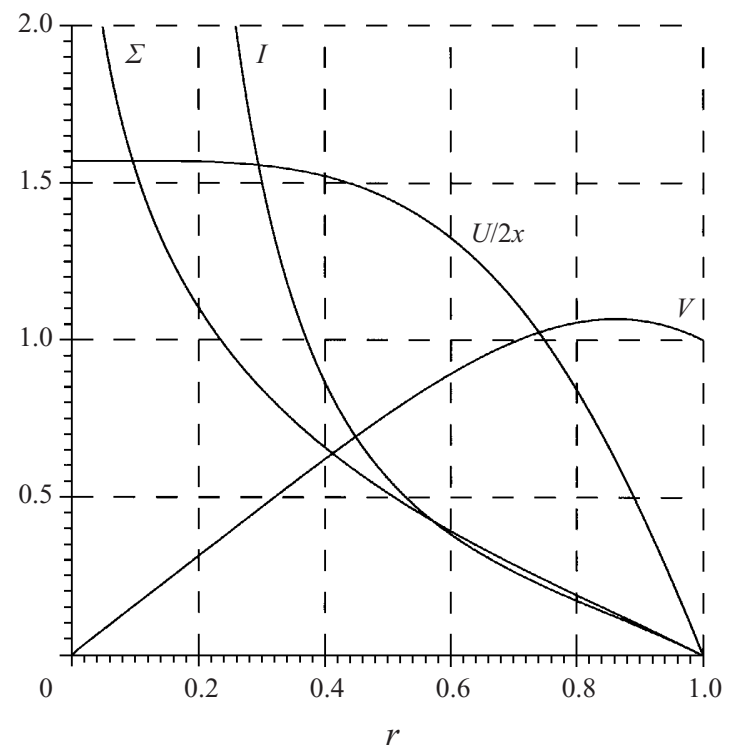

FIGURE 2. Steady axial and radial velocity profiles, $\tilde{U} /(2 x)$ and $\tilde{V}$, and the functions $\Sigma(r)$ and $I(r)$, defined in equation (62).

$$
\begin{aligned}
& U / \epsilon c_{b}=\tilde{U}=\pi x \cos \left(\frac{1}{2} \pi r^{2}\right)=x \frac{1}{r} \frac{\mathrm{d} F}{\mathrm{~d} r}, \\
& V / V_{b}=\tilde{V}=-\frac{\sin \left(\frac{1}{2} \pi r^{2}\right)}{r}=-\frac{1}{r} F(r),
\end{aligned}
$$

in terms of the coordinates $x$ and $r$ measured, respectively, in terms of the chamber length $L$ and radius $a$. The functions $\tilde{U} / 2 x$ and $\tilde{V}$ of $r$ are shown in figure 2 .

As indicated by Dunlap et al. (1979), the inviscid solution fails in the head-end region, of size $a$, of the chamber because the boundary layer on the non-burning wall separates due to an adverse radial pressure gradient, as described by Balakrishnan et al. (1992). The self-similar solution, (1)-(3), although it also fails in a region of size $a$ near the nozzle end of the chamber, is applicable to the description of the flow in most of the chamber in the cases considered here with values of the slenderness ratio, $L / a=1 / \epsilon^{\prime}$, large compared with unity.

The quasi-steady variations of pressure, temperature and density in the chamber, measured in terms of their values $p_{b}, T_{b}$ and $\rho_{b}=p_{b} / R_{g} T_{b}$ in the head-end of the chamber, are small, of the order of the square of the characteristic value $\epsilon=M_{b} / \epsilon^{\prime}$ of the Mach number of the axial flow, which we consider to be small compared with unity. These variations will not be included in (5) below, because they will not play a role in our final analysis.

The temperature $T_{b}$ of the burned gases is determined by global thermo-chemistry considerations of the quasi-steady response of the reaction layer, which also lead to a relation determining the velocity $V_{b}=M_{b} c_{b}$ of the burned gases as a function of the chamber pressure $p_{b}$. The nozzle throat area $A_{t}$ is given in terms of $V_{b}$, or $\epsilon$, and $\gamma$, the ratio of the specific heats, by the relation

$$
\frac{A_{t}}{\pi a^{2}}=2 \epsilon\left(\frac{\gamma+1}{2}\right)^{(\gamma+1) / 2(\gamma-1)}
$$


resulting from the global mass conservation in the chamber and the assumption of uniform choked flow at the throat of the nozzle. The typical values of the Mach number $M_{b}$ of the burned gases at the end of the reaction layer are in the range $10^{-3}$ to $10^{-2}$, which together with the typical small value of $\epsilon^{\prime}$ imply small values of $\epsilon$ and, hence, of the ratio $A_{t} / \pi a^{2}$ of the nozzle area to the cross-sectional area of the chamber.

\section{Formulation of the linear stability problem}

We shall write in this section the equations that describe the evolution of the linear perturbations of the basic quasi-steady flow, associated with the longitudinal acoustic waves in the chamber.

We write the fluid variables in the following form:

$$
\begin{gathered}
p / p_{b}=1+\lambda p^{\prime}, \quad T / T_{b}=1+\lambda T^{\prime}, \quad \rho / \rho_{b}=1+\lambda \rho^{\prime}, \\
u / c_{b}=\epsilon \tilde{U}+\lambda u^{\prime}, \quad v /\left(\epsilon^{\prime} c_{b}\right)=\epsilon\left(\tilde{V}+\lambda v^{\prime}\right),
\end{gathered}
$$

where the variables with tilde and primes representing, respectively, the steady flow and the oscillations, are assumed to be of order unity. The amplitude of the oscillations is measured by $\lambda \ll 1$ in our linear stability analysis, where we neglect terms not linear in $\lambda$. When $\lambda$ grows to values of order $\epsilon$, nonlinear terms affect the growth or decay of the oscillations.

The scaling used to measure the transverse velocity component of the oscillation takes into account that, due to the quasi-steady response of the gasification rate to the pressure oscillations, they are of the order $\lambda p^{\prime} V_{b}$. In the analysis we shall also consider both $\epsilon^{\prime}$ and $\epsilon$-typically of order $\epsilon^{\prime}$-to be small compared with unity, so that a multiple scales perturbation analysis will be used to describe the oscillations.

We shall begin by writing the Navier-Stokes equations in non-dimensional form using cylindrical coordinates, assuming the flow to have axial symmetry. The time is measured in terms of the acoustic time $t_{a}$, and $x$ and $r$ are measured in terms of $L$ and $a$. We introduce the definitions of (5) and (6), and neglect in the equations the terms not linear in $\lambda$. Then, we obtain the following system of equations for the oscillations:

$$
\begin{gathered}
\frac{\mathrm{D} \rho^{\prime}}{\mathrm{D} t}+\frac{\partial u^{\prime}}{\partial x}+\epsilon \frac{1}{r} \frac{\partial\left(r v^{\prime}\right)}{\partial r}=0, \\
\frac{\mathrm{D} T^{\prime}}{\mathrm{D} t}-\frac{\gamma-1}{\gamma} \frac{\mathrm{D} p^{\prime}}{\mathrm{D} t}=\frac{v}{P r} \epsilon^{3} \frac{1}{r} \frac{\partial}{\partial r}\left(r \frac{\partial T^{\prime}}{\partial r}\right), \\
\frac{\mathrm{D} u^{\prime}}{\mathrm{D} t}+\epsilon u^{\prime} \frac{\partial \tilde{U}}{\partial x}+\frac{1}{\gamma} \frac{\partial p^{\prime}}{\partial x}=v \epsilon^{3} \frac{1}{r} \frac{\partial}{\partial r}\left(r \frac{\partial u^{\prime}}{\partial r}\right), \\
\frac{\partial p^{\prime}}{\partial r}=0, \\
p^{\prime}-\rho^{\prime}-T^{\prime}=0 .
\end{gathered}
$$

In (7)-(11) $P r=v_{b} / \alpha_{b}$ is the Prandtl number, of order unity, and $v=v_{b} / a V_{b} \epsilon^{2}=$ $v_{b} / \epsilon^{\prime} \epsilon^{3} a c_{b}$ is the non-dimensional kinematic viscosity, defined so that when $v$ is of order unity, corresponding to the distinguished limit of our analysis, the vorticity waves travel radially distances of the order of the chamber radius before they are 
damped. The material derivative in the above equations takes the form

$$
\frac{\mathrm{D}}{\mathrm{D} t}=\frac{\partial}{\partial t}+\epsilon \tilde{U} \frac{\partial}{\partial x}+\epsilon \tilde{V} \frac{\partial}{\partial r} .
$$

We have neglected in these equations all the terms of order $\epsilon^{2}$ or higher, except for the viscous and heat transfer terms, apparently of order $\epsilon^{3}$, appearing in (8) and (9). These terms, which are responsible for the attenuation of the entropy and vorticity waves, are in fact of order $\epsilon$ due to the rapid radial variations, with scale $\epsilon$, of $T^{\prime}$ and $u^{\prime}$. The other terms left out of the system of equations (7)-(11) do not enter into our linear stability analysis when $\epsilon$ and $\epsilon^{\prime}$ are both small.

The equations are complemented with the following boundary conditions to be used in our asymptotic analysis:

(i) At $r=0$ the symmetry condition,

$$
v^{\prime}=0 .
$$

(ii) At $r=1$ matching conditions with the thin gas-phase reaction layer, no-slip condition

$$
u^{\prime}=0
$$

constant temperature of the combustion gases

$$
T^{\prime}=0,
$$

solid-propellant admittance condition

$$
v^{\prime}=-A_{b} p^{\prime}
$$

This relation, where the solid propellant admittance $A_{b}$, of order unity, is a function of the oscillation frequency and of the physical properties of the propellant and the combustion products, results from the analysis of the thin reaction layer, which also leads to $T^{\prime}=0$. For the dependence of $A_{b}$ on the oscillation frequency see Clavin \& Lazimi (1992). The effect of the oscillating component $v^{\prime}$ of the radial velocity was neglected in the analysis of Majdalani \& Van Moorhem (1997), but it plays an essential role in our analysis.

(iii) At $x=0$, the non-burning head-end of the chamber, we will allow the radial velocity to be non-zero in our large Reynolds number analysis, which does not include a description of the structure of the head-end boundary layer, but we shall enforce

$$
u^{\prime}=0 \text {. }
$$

The description of the boundary conditions at the chamber exit, where the parameter $\epsilon^{\prime}$ also plays a role, will be postponed to $\S 5$.

\section{Multiple scale analysis}

As indicated above, and as shown in previous analysis and experiments (see Flandro 1989, 1995; Avalon \& Comas 1991; Vuillot 1991; Vuillot \& Avalon 1991; Lupoglazoff \& Vuillot 1991), the acoustic oscillations in the chamber lead to vorticity as well as entropy waves. These have a wavelength, variable with $r$, of order $l_{0}=\epsilon a$, and their amplitude will change, due to viscous and heat conduction damping and also due to flow field interaction, at radial distances of order $a$ in the distinguished regime that we shall analyse with a multiple scale technique (see for example Bender \& Orszag 
1978). Because the fast oscillations occur in the radial direction we shall introduce, in addition to the slow radial variable $r$, the following fast radial variable

$$
\xi=\frac{1}{\epsilon} \int_{1}^{r} \frac{\mathrm{d} r}{\tilde{V}}=-\frac{1}{\epsilon} \frac{1}{\pi} \ln \left[\tan \left(\frac{1}{4} \pi r^{2}\right)\right]=\frac{1}{\epsilon} \Sigma(r),
$$

which is the time, in units of the acoustic time, of travel of a fluid particle from $r=1$ to $r . \Sigma / \epsilon$, where $\Sigma(r)$ is plotted in figure 2, characterizes the phase of the vorticity and entropy oscillations of the fluid particles. We shall also introduce two time variables: the fast time $t$, based on the acoustic time, and the slow time variable $\tau=\epsilon t$, based on the residence time $a / V_{b}$, which, in our distinguished regime, is of the order of the attenuation time, $t_{a t}$, of the vorticity and entropy waves.

With the introduction of the new fast and slow variables the radial and time derivatives, appearing in (7)-(10), transform to

$$
\frac{\partial}{\partial r} \rightarrow \frac{\partial}{\partial r}+\frac{1}{\epsilon} \frac{1}{\tilde{V}} \frac{\partial}{\partial \xi}, \quad \frac{\partial}{\partial t} \rightarrow \frac{\partial}{\partial t}+\epsilon \frac{\partial}{\partial \tau},
$$

when $\xi$ and $\tau$ are considered as independent variables, together with $r, x$ and $t$. The final form taken by the equations is

$$
\begin{gathered}
\frac{\partial \rho^{\prime}}{\partial t}+\frac{\partial \rho^{\prime}}{\partial \xi}+\epsilon\left[\frac{\partial \rho^{\prime}}{\partial \tau}+\tilde{U} \frac{\partial \rho^{\prime}}{\partial x}+\tilde{V} \frac{\partial \rho^{\prime}}{\partial r}\right]+\frac{\partial u^{\prime}}{\partial x}+\frac{1}{\tilde{V}} \frac{\partial v^{\prime}}{\partial \xi}+\epsilon \frac{1}{r} \frac{\partial\left(r v^{\prime}\right)}{\partial r}=0, \\
\frac{\partial T^{\prime}}{\partial t}+\frac{\partial T^{\prime}}{\partial \xi}+\epsilon\left[\frac{\partial T^{\prime}}{\partial \tau}+\tilde{U} \frac{\partial T^{\prime}}{\partial x}+\tilde{V} \frac{\partial T^{\prime}}{\partial r}\right]-\frac{\gamma-1}{\gamma}\left[\frac{\partial p^{\prime}}{\partial t}+\epsilon \frac{\partial p^{\prime}}{\partial \tau}+\epsilon \tilde{U} \frac{\partial p^{\prime}}{\partial x}\right] \\
=\epsilon \frac{v}{\operatorname{Pr}} \frac{1}{\tilde{V}^{2}} \frac{\partial^{2} T^{\prime}}{\partial \xi^{2}}, \\
\frac{\partial u^{\prime}}{\partial t}+\frac{\partial u^{\prime}}{\partial \xi}+\epsilon\left[\frac{\partial u^{\prime}}{\partial \tau}+\tilde{U} \frac{\partial u^{\prime}}{\partial x}+\tilde{V} \frac{\partial u^{\prime}}{\partial r}+u^{\prime} \frac{\partial \tilde{U}}{\partial x}\right]+\frac{1}{\gamma} \frac{\partial p^{\prime}}{\partial x}=\epsilon v \frac{1}{\tilde{V}^{2}} \frac{\partial^{2} u^{\prime}}{\partial \xi^{2}}, \\
\frac{\partial p^{\prime}}{\partial r}+\frac{1}{\epsilon} \frac{1}{\tilde{V}} \frac{\partial p^{\prime}}{\partial \xi}=0,
\end{gathered}
$$

while (11) remains unchanged. Notice that in the distinguished regime that we analyse, $v_{b} / v_{c}=v$ of order unity, the viscous and heat conduction terms are of order $\epsilon$.

\subsection{Perturbation scheme}

Regular multiple scale perturbation techniques are used to solve the problem. The acoustic fluctuations, $p^{\prime}, T^{\prime}, \rho^{\prime}, u^{\prime}$ and $v^{\prime}$, are expanded in perturbation series in powers of the small parameter $\epsilon$, of the form

$$
()^{\prime}=()_{0}+\epsilon()_{1}+\cdots,
$$

where the coefficients in the expansion, for example $u_{0}$ and $u_{1}$ in the expansion for $u^{\prime}$, are bounded functions, of order unity, of the variables $t, \tau, \xi, r$ and $x$. These, in the linear acoustic analysis that we carry out with multiple scales, are periodic functions of $t$ and $\xi$. For the solid-propellant admittance function we write

$$
A_{b}=A_{b_{0}}+\epsilon A_{b_{1}}+\cdots .
$$

If these expansions are inserted into (20)-(23), (11) and boundary conditions (13)-(17), the following system of equations is generated: 
At $O(1)$ :

$$
\begin{gathered}
-\frac{\partial v_{0}}{\partial \xi}=\tilde{V}\left[\frac{\partial u_{0}}{\partial x}+\frac{\partial \rho_{0}}{\partial t}+\frac{\partial \rho_{0}}{\partial \xi}\right], \\
\frac{\partial T_{0}}{\partial t}+\frac{\partial T_{0}}{\partial \xi}-\frac{\gamma-1}{\gamma} \frac{\partial p_{0}}{\partial t}=0 \\
\frac{\partial u_{0}}{\partial t}+\frac{\partial u_{0}}{\partial \xi}+\frac{1}{\gamma} \frac{\partial p_{0}}{\partial x}=0 \\
\frac{\partial p_{0}}{\partial r}=\frac{\partial p_{0}}{\partial \xi}=0 \\
p_{0}-\rho_{0}-T_{0}=0
\end{gathered}
$$

with the boundary conditions:

at $r=0$ :

$$
v_{0}=0
$$

at $r=1$ :

$$
\begin{gathered}
u_{0}=0, \quad T_{0}=0, \\
v_{0}=-A_{b_{0}} p_{0} ;
\end{gathered}
$$

at $x=0$ :

$$
u_{0}=0
$$

At $O(\epsilon)$ :

$$
\begin{gathered}
-\frac{\partial v_{1}}{\partial \xi}=\tilde{V}\left[\frac{\partial u_{1}}{\partial x}+\frac{\partial \rho_{1}}{\partial t}+\frac{\partial \rho_{1}}{\partial \xi}+\frac{v_{0}}{r}+\frac{\partial v_{0}}{\partial r}+\frac{\partial \rho_{0}}{\partial \tau}+\tilde{U} \frac{\partial \rho_{0}}{\partial x}+\tilde{V} \frac{\partial \rho_{0}}{\partial r}\right] \\
\frac{\partial T_{1}}{\partial t}+\frac{\partial T_{1}}{\partial \xi}-\frac{\gamma-1}{\gamma}\left[\frac{\partial p_{1}}{\partial t}+\frac{\partial p_{0}}{\partial \tau}+\tilde{U} \frac{\partial p_{0}}{\partial x}\right]+\frac{\partial T_{0}}{\partial \tau}+\tilde{U} \frac{\partial T_{0}}{\partial x}+\tilde{V} \frac{\partial T_{0}}{\partial r}=\frac{v}{P r} \frac{1}{\tilde{V}^{2}} \frac{\partial^{2} T_{0}}{\partial \xi^{2}} \\
\frac{\partial u_{1}}{\partial t}+\frac{\partial u_{1}}{\partial \xi}+\frac{1}{\gamma} \frac{\partial p_{1}}{\partial x}+\frac{\partial u_{0}}{\partial \tau}+\tilde{U} \frac{\partial u_{0}}{\partial x}+\tilde{V} \frac{\partial u_{0}}{\partial r}+u_{0} \frac{\partial \tilde{U}}{\partial x}=v \frac{1}{\tilde{V}^{2}} \frac{\partial^{2} u_{0}}{\partial \xi^{2}} \\
\frac{\partial p_{1}}{\partial r}=\frac{\partial p_{1}}{\partial \xi}=0 \\
p_{1}-\rho_{1}-T_{1}=0
\end{gathered}
$$

with the boundary conditions:

at $r=0$ :

$$
v_{1}=0,
$$

at $r=1$ :

at $x=0$ :

$$
\begin{gathered}
u_{1}=0, \quad T_{1}=0, \\
v_{1}=-A_{b_{1}} p_{0}-A_{b_{0}} p_{1},
\end{gathered}
$$

$$
u_{1}=0 .
$$




\subsection{Acoustic flow field calculations}

We are considering here oscillations of low frequency, associated with acoustic longitudinal waves. Consequently, as will be shown below, the pressure perturbations of zeroth-order correspond to axial plane standing waves. When describing the linear acoustic oscillations we can assume a harmonic dependence of the time variables of the form $\mathrm{e}^{\mathrm{i} \omega t+\sigma \tau}$. The fast time dependence $\mathrm{e}^{\mathrm{i} \omega t}$ involves the real dimensionless angular frequency $\omega$, of order unity. The growth, or decay, of the amplitude is slow, represented by the factor $\mathrm{e}^{\sigma \tau}$, involving the long time scale. The stability exponent $\sigma$ is a complex number, $\sigma=\sigma_{R}+\mathrm{i} \sigma_{I}$; the sign of the real part of $\sigma$ gives the growth rate of the oscillations ( $\sigma_{R}>0$ implying instability); the imaginary part of $\sigma$ represents a frequency shift, a correction to $\omega$, of order $\epsilon$. Both $\omega$ and $\sigma$ are unknown eigenvalues of the problem, which must be determined as part of the solution. Equations (29) and (38) show that up to the order considered, $p^{\prime}$ is independent of $\xi$ and $r$. Then, the structure of the zeroth-order system (27)-(30), where due to our choice of $\xi$ the mean flow does not appear, allows us to write the solution in the form

$$
\begin{gathered}
p_{0}=A(x) \mathrm{e}^{\mathrm{i} \omega t+\sigma \tau}, \\
T_{0}=\frac{\gamma-1}{\gamma} A(x) \mathrm{e}^{\mathrm{i} \omega t+\sigma \tau}+\bar{T}_{0}(x, r) \mathrm{e}^{\mathrm{i} \omega(t-\xi)+\sigma \tau}, \\
\rho_{0}=\frac{1}{\gamma} A(x) \mathrm{e}^{\mathrm{i} \omega t+\sigma \tau}-\bar{T}_{0}(x, r) \mathrm{e}^{\mathrm{i} \omega(t-\xi)+\sigma \tau} \\
u_{0}=\frac{\mathrm{i}}{\gamma \omega} A_{x}(x) \mathrm{e}^{\mathrm{i} \omega t+\sigma \tau}+\bar{u}_{0}(x, r) \mathrm{e}^{\mathrm{i} \omega(t-\xi)+\sigma \tau},
\end{gathered}
$$

where the temperature, density and velocity fields are shown decomposed into two parts. The first part, as well as $p_{0}$, corresponds to the isentropic irrotational classical acoustic oscillations, $\hat{T}_{0}^{\prime}, \hat{\rho}_{0}^{\prime}$ and $\hat{u}_{0}^{\prime}$. The second part represents the entropy and vorticity waves, $\bar{T}_{0}^{\prime}, \bar{\rho}_{0}^{\prime}$ and $\bar{u}_{0}^{\prime}$, associated with the isothermal and the no-slip condition $T_{0}=0$ and $u_{0}=0$ at the propellant surface $r=1, \xi=0$, so that $\bar{T}_{0}(x, 1)=$ $-A(x)(\gamma-1) / \gamma$ and $\bar{u}_{0}(x, 1)=-A_{x}(x) \mathrm{i} / \gamma \omega$. Note that the entropy variations from the basic state, when scaled with $c_{v}$, are given by $s^{\prime}=T^{\prime}-(\gamma-1) \rho^{\prime}$, and in a first approximation are equal to $\gamma$ times the last term in (45). The entropy and vorticity waves have the phase $i \omega(t-\xi)$, corresponding to the time $(t-\xi)$ of departure of the particle from the propellant surface, to be compared with the phase i $\omega t$ of the acoustic waves. The entropy $s^{\prime}$ and vorticity $\omega_{\theta}^{\prime}$, measured with $c_{v}$ and $c_{b} / \epsilon a$ respectively, are given, for the zeroth-order solution, by

$$
\begin{gathered}
s_{0}=\bar{s}_{0}(x, r) \mathrm{e}^{\mathrm{i} \omega(t-\xi)+\sigma \tau}=\gamma \bar{T}_{0}(x, r) \mathrm{e}^{\mathrm{i} \omega(t-\xi)+\sigma \tau}, \\
\omega_{\theta_{0}}=\bar{\omega}_{\theta_{0}}(x, r) \mathrm{e}^{\mathrm{i} \omega(t-\xi)+\sigma \tau}=-\frac{1}{\tilde{V}} \bar{u}_{0}(x, r) \mathrm{e}^{\mathrm{i} \omega(t-\xi)+\sigma \tau} .
\end{gathered}
$$

The amplitude $A(x)$ of the acoustic pressure oscillations and the amplitudes, $\bar{T}_{0}$ and $\bar{u}_{0}$, of the entropy and vorticity waves can be determined by eliminating secular terms and using the solvability conditions of (26) and of (36)-(37), corresponding to the terms of order $\epsilon$. The resulting equations for $v_{0}, T_{1}$ and $u_{1}$ are

$$
-\frac{\partial v_{0}}{\partial \xi}-\tilde{V} \frac{\partial \bar{u}_{0}}{\partial x} \mathrm{e}^{\mathrm{i} \omega(t-\xi)+\sigma \tau}=\tilde{V} \frac{\mathrm{i}}{\gamma \omega}\left(A_{x x}+\omega^{2} A\right) \mathrm{e}^{\mathrm{i} \omega t+\sigma \tau},
$$


Longitudinal acoustic instabilities in solid propellant rockets

$$
\begin{gathered}
\frac{\partial T_{1}}{\partial t}+\frac{\partial T_{1}}{\partial \xi}-\frac{\gamma-1}{\gamma} \frac{\partial p_{1}}{\partial t}=-\left[\left(\sigma+\frac{v \omega^{2}}{P r} \frac{1}{\tilde{V}^{2}}\right) \bar{T}_{0}+\tilde{U} \frac{\partial \bar{T}_{0}}{\partial x}+\tilde{V} \frac{\partial \bar{T}_{0}}{\partial r}\right] \mathrm{e}^{\mathrm{i} \omega(t-\xi)+\sigma \tau} \\
\frac{\partial u_{1}}{\partial t}+\frac{\partial u_{1}}{\partial \xi}+\frac{1}{\gamma} \frac{\partial p_{1}}{\partial x}+\frac{\mathrm{i}}{\gamma \omega}\left(\sigma A_{x}+\tilde{U} A_{x x}+\frac{\partial \tilde{U}}{\partial x} A_{x}\right) \mathrm{e}^{\mathrm{i} \omega t+\sigma \tau} \\
=-\left[\left(\sigma+\frac{\partial \tilde{U}}{\partial x}+v \omega^{2} \frac{1}{\tilde{V}^{2}}\right) \bar{u}_{0}+\tilde{U} \frac{\partial \bar{u}_{0}}{\partial x}+\tilde{V} \frac{\partial \bar{u}_{0}}{\partial r}\right] \mathrm{e}^{\mathrm{i} \omega(t-\xi)+\sigma \tau}
\end{gathered}
$$

We eliminate secular terms by equating the terms on the right-hand sides of (50)-(52) to zero, thus obtaining the desired amplitude equations

$$
\begin{gathered}
A_{x x}+\omega^{2} A=0, \\
\left(\sigma+\frac{v \omega^{2}}{P r} \frac{1}{\tilde{V}^{2}}\right) \bar{T}_{0}+\tilde{U} \frac{\partial \bar{T}_{0}}{\partial x}+\tilde{V} \frac{\partial \bar{T}_{0}}{\partial r}=0, \\
\left(\sigma+\frac{\partial \tilde{U}}{\partial x}+v \omega^{2} \frac{1}{\tilde{V}^{2}}\right) \bar{u}_{0}+\tilde{U} \frac{\partial \bar{u}_{0}}{\partial x}+\tilde{V} \frac{\partial \bar{u}_{0}}{\partial r}=0 .
\end{gathered}
$$

Solving (53), with the boundary condition (34), together with (28), yields

$$
A(x)=\cos (\omega x) \text {, }
$$

where, with the appropriate choice of $\lambda, A(0)$ has been normalized to 1 . Thus, the zeroth-order pressure oscillation is given by

$$
p_{0}=\cos (\omega x) \mathrm{e}^{\mathrm{i} \omega t+\sigma \tau},
$$

corresponding to classical acoustics. We anticipate here the result, obtained later in $\S 5$, that, because $A_{t} / a^{2}=O(\epsilon)$, the pure acoustic component of $u_{0}$ is zero at $x=1$, so that $A_{x}(1)=0$ and $\sin \omega=0$. So that $\omega=n \pi$ with $n$ an integer.

To compute $\bar{T}_{0}$ and $\bar{u}_{0}$ we shall replace the variables $r$ and $x$ by $r$ and the stream function $\tilde{\Psi}=x F(r)$ of the steady flow. Equations (54) and (55) transform to the system

$$
\begin{gathered}
\left(\sigma+\frac{v \omega^{2}}{P r} \frac{r^{2}}{F^{2}}\right) \bar{T}_{0}-\frac{F}{r} \frac{\partial \bar{T}_{0}}{\partial r}=0, \\
\left(\sigma+\frac{1}{r} \frac{\mathrm{d} F}{\mathrm{~d} r}+v \omega^{2} \frac{r^{2}}{F^{2}}\right) \bar{u}_{0}-\frac{F}{r} \frac{\partial \bar{u}_{0}}{\partial r}=0,
\end{gathered}
$$

which involves only derivatives with respect to $r$ with $\tilde{\Psi}$ fixed. We can solve these equations, accounting for the boundary conditions (32), to calculate $\bar{T}_{0}$ and $\bar{u}_{0}$-and thus the amplitudes of the entropy and vorticity waves - given by

$$
\begin{gathered}
\bar{T}_{0}(x, r)=-\frac{\gamma-1}{\gamma} \cos [\omega x F(r)] \exp \left[-\frac{v \omega^{2}}{P r} I(r)-\sigma \Sigma(r)\right], \\
\bar{u}_{0}(x, r)=\frac{\mathrm{i}}{\gamma} \sin [\omega x F(r)] F(r) \exp \left[-v \omega^{2} I(r)-\sigma \Sigma(r)\right],
\end{gathered}
$$

where the functions $\Sigma(r)$ and $I(r)$, plotted in figure 2, are given by

$$
\Sigma(r)=-\int_{1}^{r} \frac{r \mathrm{~d} r}{F(r)}, \quad I(r)=-\int_{1}^{r} \frac{r^{3}}{F^{3}(r)} \mathrm{d} r .
$$


Integration of (50), taking into account (53), yields

$$
v_{0}=\left[-\frac{\mathrm{i}}{\omega} \tilde{V} \frac{\partial \bar{u}_{0}}{\partial x} \mathrm{e}^{-\mathrm{i} \omega \xi}+N(x, r)\right] \mathrm{e}^{\mathrm{i} \omega t+\sigma \tau}
$$

where $N(x, r)$ can be obtained from the solvability conditions of the second-order problem. Using (38), the first-order correction of the pressure oscillation can also be written in the form

$$
p_{1}=B(x) \mathrm{e}^{\mathrm{i} \omega t+\sigma \tau} \text {. }
$$

The first-order corrections of the temperature and axial velocity component, given by the solution (36) and (37) without secular terms, take the form

$$
\begin{gathered}
T_{1}=\frac{\gamma-1}{\gamma} B(x) \mathrm{e}^{\mathrm{i} \omega t+\sigma \tau}+\bar{T}_{1}(x, r) \mathrm{e}^{\mathrm{i} \omega(t-\xi)+\sigma \tau}, \\
u_{1}=\frac{\mathrm{i}}{\gamma \omega}\left[B_{x}(x)+\frac{\mathrm{i}}{\omega}\left(\sigma A_{x}+\tilde{U} A_{x x}+\frac{\partial \tilde{U}}{\partial x} A_{x}\right)\right] \mathrm{e}^{\mathrm{i} \omega t+\sigma \tau}+\bar{u}_{1}(x, r) \mathrm{e}^{\mathrm{i} \omega(t-\xi)+\sigma \tau},
\end{gathered}
$$

where $T_{1}$ and $u_{1}$ are written in terms of their acoustic parts $\hat{T}_{1}^{\prime}$ and $\hat{u}_{1}^{\prime}$ represented by the first terms on the right-hand sides of (65) and (66) and the entropy and vorticity parts, given by the last terms of these equations. The density correction $\rho_{1}$ is obtained from (39). To calculate $v_{1}$ we use (35) with the previous results to distinguish secular from non-secular terms. Finally, the equation for $v_{1}$ takes the form

$$
\begin{aligned}
-\frac{1}{\tilde{V}} \frac{\partial v_{1}}{\partial \xi}+\frac{\mathrm{i}}{\omega}\left[\mathrm{i} \omega \frac{\partial \bar{u}_{1}}{\partial x}-\frac{\tilde{V}}{r} \frac{\partial \bar{u}_{0}}{\partial x}-\frac{\partial}{\partial r}\left(\tilde{V} \frac{\partial \bar{u}_{0}}{\partial x}\right)\right] \mathrm{e}^{\mathrm{i} \omega(t-\xi)+\sigma \tau}-\frac{v \omega^{2}}{P r} \frac{1}{\tilde{V}^{2}} \bar{T}_{0} \mathrm{e}^{\mathrm{i} \omega(t-\xi)+\sigma \tau} \\
=\left[\frac{\mathrm{i}}{\gamma \omega}\left[B_{x x}+\omega^{2} B+\frac{\mathrm{i}}{\omega}\left(\sigma A_{x x}+2 \tilde{U}_{x} A_{x x}+\tilde{U} A_{x x x}\right)\right]\right. \\
\left.+\frac{N}{r}+\frac{\partial N}{\partial r}+\frac{\sigma}{\gamma} A+\frac{1}{\gamma} \tilde{U} A_{x}\right] \mathrm{e}^{\mathrm{i} \omega t+\sigma \tau} .
\end{aligned}
$$

We eliminate the secular terms by equating the right-hand side of this equation to zero, and thus obtain the equation for $N(x, r)$, whose general solution is

$$
N(x, r)=\frac{1}{r} G(x)+\frac{r}{2 \mathrm{i} \omega \gamma}\left(\omega^{2} B+B_{x x}-2 \mathrm{i} \omega \sigma A\right)-\frac{2}{\gamma}\left(x A_{x}+A\right) \frac{F(r)}{r} .
$$

The boundary condition (31) for $v_{0}$ implies that $N(x, 0)=0$ and hence $G(x)=0$. At this point, using the pressure-coupled response of the solid propellant for $v_{0}$, relation (33), the equation for $B(x)$ is generated,

$$
\omega^{2} B+B_{x x}=2 \mathrm{i} \omega\left[\left(-\gamma A_{b_{0}}+\sigma+3\right) \cos (\omega x)-2 \omega x \sin (\omega x)\right],
$$

and, when used in (68), we obtain

$$
N(x, r)=-\frac{2}{\gamma}[\cos (\omega x)-\omega x \sin (\omega x)] \frac{F(r)}{r}+\frac{r}{\gamma}\left[\left(-\gamma A_{b_{0}}+3\right) \cos (\omega x)-2 \omega x \sin (\omega x)\right] .
$$

If we integrate (69) with the normalization condition $B_{x}(0)=0$ resulting from (43),

$$
B(x)=\delta \cos (\omega x)+\mathrm{i} \omega x^{2} \cos (\omega x)+\mathrm{i}\left[-\gamma A_{b_{0}}+\sigma+2\right] x \sin (\omega x),
$$

with $\delta$ a constant. In the following we shall write $\delta=0$, based on our choice of the normalization of the oscillation amplitude of the pressure at $x=0$. 


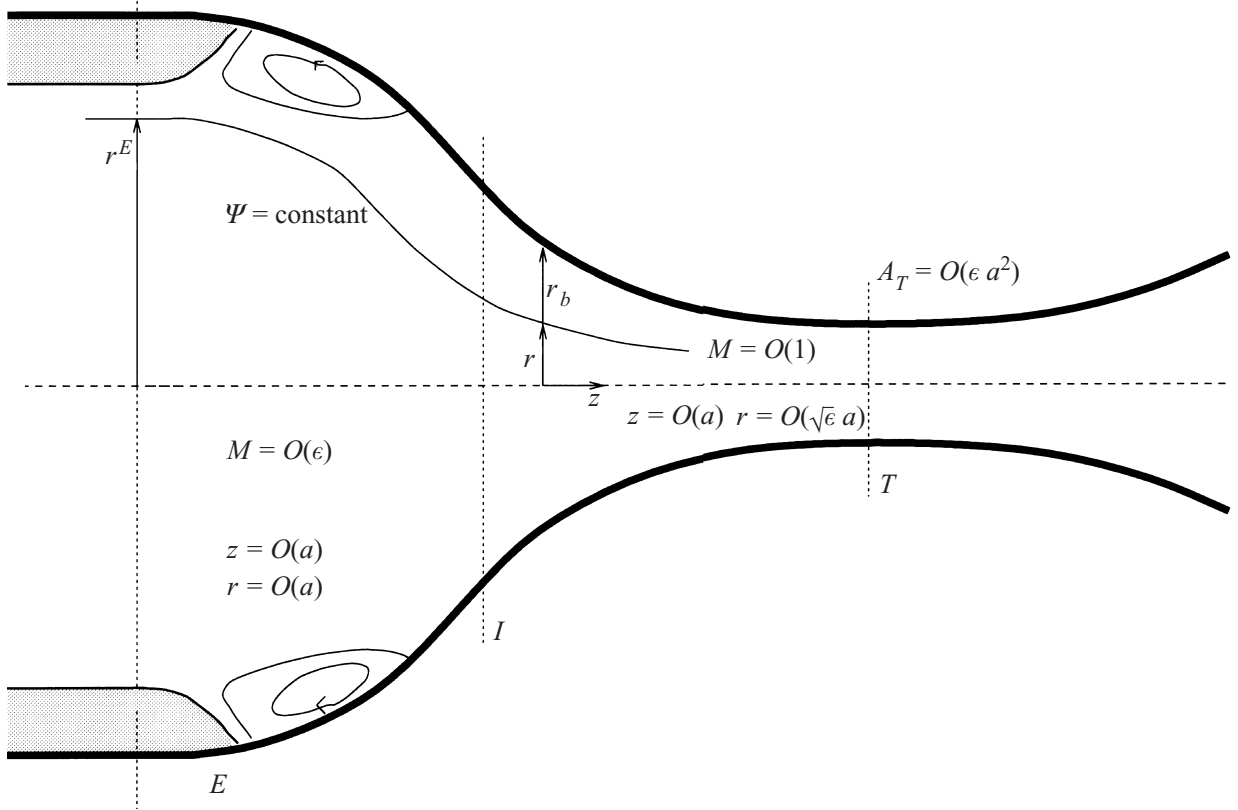

FIGURE 3. The entrance and throat regions of the nozzle.

We thus have, aside from the values of $\omega$ and $\sigma$, a complete description of the zeroth-order flow field, and also $p_{1}$ and the acoustic parts of $u_{1}$ and $T_{1}$.

\section{Nozzle analysis}

Up to now we have not used the boundary conditions at the chamber exit, which are needed to determine the angular frequency $\omega$, as well as the growth rate (real part of $\sigma$ ) and the small correction to the frequency (imaginary part of $\sigma$ ). These boundary conditions, representing the nozzle admittance function, depend on how the non-steady flow evolves through the nozzle. If we were to consider the flow toward supersonic velocities in the nozzle as irrotational, isentropic and quasi-steady, then the flow would be choked with the sonic condition at the throat. However, due to the time variations and the vorticity and entropy waves of the flow entering the nozzle the choking conditions at the throat have to be described. Thus, we shall account here for non-steady effects in the evolution of the flow field through the nozzle, as done previously by Crocco \& Cheng (1956) and Crocco \& Sirignano (1967), and also for the possible effects, not considered previously, of the entropy and vorticity waves in this flow.

We shall take advantage in our analysis of the fact that the throat area is $\epsilon$ times smaller than the cross-sectional area of the chamber, and divide the nozzle into the two regions sketched in figure 3, with steady-flow Mach numbers of order $\epsilon$ and 1 . The first region is at the entrance of the nozzle, with cross-sectional area of order $a^{2}$, where the velocity of the mean flow is still, of the order $\epsilon c_{b}$, small compared with the speed of sound $c_{b}$. We shall consider that the characteristic length of this region is of order $a$, small compared with $L$. The volume $\Omega$ of this region is $\Omega=\beta^{\prime} \pi a^{3}$, with $\beta^{\prime}=O(1)$, small compared with the chamber volume $\pi a^{2} L$. The second region of the nozzle is the throat region, with slowly varying cross-sectional area of order $\epsilon \pi a^{2}$, so 
that the quasi-steady velocity is of order $c_{b}$. The flow in this region can be treated as quasi-steady, because the residence time is much shorter than the acoustic time and, then, the stagnation enthalpy and entropy, as well as the ratio of the vorticity to the product of the density and the local radius, are, in first approximation, conserved along each streamline. The separate simplified analysis to be carried out for the two regions has a common domain of validity in the intermediate region, represented by a station $I$, so that the flow in the low Mach number region can be matched with that of the quasi-steady region.

For the analysis of the flow in these regions, we need to solve the conservation equations with a multiple scale technique, in a form similar to that used in $\S 4$. The fluid variables to be determined in the nozzle are the steady flow values of the density $\tilde{\rho}$, pressure $\tilde{p}$ and temperature $\tilde{T}$, measured in terms of $\rho_{b}, p_{b}$ and $T_{b}$, respectively, the steady velocities $\tilde{u}$ and $\tilde{v}$, based on $c_{b}$, and the non-steady perturbations $\lambda \rho^{\prime}, \lambda p^{\prime}$, $\lambda T^{\prime}$ and $\lambda u^{\prime}$, measured in terms of $\rho_{b}, p_{b}, T_{b}$ and $c_{b}$, which are the orders of these variables in the quasi-steady region. In the following the oscillations will be separated into their acoustic contributions, denoted with a hat, and the rapid radially varying contributions, denoted with an overbar. Thus, for example $\rho^{\prime}=\hat{\rho}^{\prime}+\bar{\rho}^{\prime}$.

\subsection{Low Mach number entrance region of the nozzle}

In the entrance region of the nozzle the mean flow values of $\tilde{u}$ and $\tilde{v}$ are of order $\epsilon$, so that in this region

$$
\tilde{u} / \epsilon=\tilde{u}_{1} \quad \text { and } \quad \tilde{v} / \epsilon=\tilde{v}_{1}
$$

are of order unity.

The overall mass conservation equation, written below as (73), will also show that the acoustic part $\hat{u}^{\prime}$ of the velocity oscillations $u^{\prime}$ in this entrance region is also of order $\epsilon$. On the other hand, the vorticity part of the velocity is, as in the exit section of the chamber, of order unity, so that according to the momentum equation, the spatial variations in this region of the pressure oscillations from the value $p_{0_{E}}$, given by (57), in the exit section of the chamber are of order $\epsilon^{2}$.

We can write, in non-dimensional form, the mass conservation equation for the low Mach number region between the sections $E$, at the end of the chamber, and $I$, as

$$
\epsilon^{\prime} \int_{\tilde{\Omega}} \frac{\partial \rho^{\prime}}{\partial t} \mathrm{~d} \tilde{\Omega}=G_{E}^{\prime}-G_{I}^{\prime},
$$

where $\tilde{\Omega}$ is the volume of the entrance region, measured in terms of $a^{3}$, and $\lambda G_{E}^{\prime}$ and $\lambda G_{I}^{\prime}$ are the oscillating parts of the mass fluxes, given by

$$
\begin{aligned}
G_{E}^{\prime} & =\int_{S_{E}}\left(\tilde{\rho} u^{\prime}+\rho^{\prime} \tilde{u}\right)_{E} \mathrm{~d} S_{E}, \\
G_{I}^{\prime} & =\int_{S_{I}}\left(\tilde{\rho} u^{\prime}+\rho^{\prime} \tilde{u}\right)_{I} \mathrm{~d} S_{I},
\end{aligned}
$$

where the integrals are taken over the cross-sections $S_{E}$ and $S_{I}$, measured in terms of $a^{2}$.

In the throat region, where the Mach number is of order 1, the flow is quasi-steady, and so we can write

$$
G_{I}^{\prime}=G_{T}^{\prime}=\int_{S_{T}}\left(\tilde{\rho} u^{\prime}+\rho^{\prime} \tilde{u}\right)_{T} \mathrm{~d} S_{T},
$$

neglecting terms of order higher than $\epsilon$ that will not be considered in the analysis 
that follows. To obtain the value of $G_{T}^{\prime}$, of order $\epsilon$ because $S_{T}=O(\epsilon)$, we have to carry out an analysis for the quasi-steady region of the nozzle in order to calculate $u^{\prime}$ and $\rho^{\prime}$ at the nozzle throat. However, we have now reasons, based on the orders of magnitude of the terms in (73), to state that $G_{E}^{\prime}$ is $O(\epsilon)$. Although the non-acoustic part of the velocity oscillations are of order unity, their contribution to $G_{E}^{\prime}$ is of order $\epsilon$ due to their rapidly radially oscillating character. Then the acoustic part of the oscillating velocity, must be of order $\epsilon$ in the entrance section $E$, and thus we may now conclude that $A_{x}(0)=0$, or

$$
\sin \omega=0,
$$

providing the first approximation for the frequency

$$
\omega=\omega_{n}=n \pi,
$$

where $n$ is an integer, corresponding to the longitudinal acoustic modes in the chamber.

$G_{E}^{\prime}$ is computed from the solution obtained, at $x=1$, in the chamber analysis, so that $\tilde{\rho}=1$ and $\tilde{u}=\epsilon r^{-1} \mathrm{~d} F / \mathrm{d} r$. Thus

$$
G_{E}^{\prime}=\int_{0}^{1}\left(\left[\bar{u}_{0}\right]_{x=1} \mathrm{e}^{\mathrm{i} \omega t+\sigma \tau-\mathrm{i} \omega \xi}+\epsilon\left[\hat{u}_{1}^{\prime}+\hat{\rho}_{0}^{\prime} \frac{1}{r} \frac{\mathrm{d} F}{\mathrm{~d} r}\right]_{x=1}\right) 2 \pi r \mathrm{~d} r,
$$

where we have left out other terms leading to contributions of order $\epsilon^{2}$ or higher.

The first integral, aside from the factor $\mathrm{e}^{\mathrm{i} \omega t+\sigma \tau}$, is

$$
\begin{aligned}
J & =\int_{0}^{1} \frac{\mathrm{i}}{\gamma} \sin [\omega F(r)] F(r) \exp \left[-v \omega^{2} I(r)-\sigma \Sigma(r)\right] \exp \left(-\mathrm{i} \omega \frac{1}{\epsilon} \Sigma(r)\right) 2 \pi r \mathrm{~d} r \\
& =\int_{0}^{1} L(r) \exp \left(-\mathrm{i} \omega \frac{1}{\epsilon} \Sigma(r)\right) \mathrm{d} r,
\end{aligned}
$$

where $L$ and $\Sigma$ are smooth functions of $r$, and $\Sigma(r)$ is strictly monotone. Using integration by parts, taking into account (18) and leaving out terms of order $\epsilon^{2}$,

$$
J=\epsilon\left[-L(r) \frac{\tilde{V}}{\mathrm{i} \omega} \exp \left(-\mathrm{i} \omega \frac{1}{\epsilon} \Sigma(r)\right)\right]_{0}^{1}=-\epsilon L(1) \frac{\mathrm{i}}{\omega}=\epsilon \frac{2 \pi}{\gamma \omega} \sin \omega,
$$

because $\tilde{V}(0)=0$. We find the same lowering of the order in $\epsilon$ when evaluating other integrals of the same type representing the effects of the vorticity and entropy waves. Their contribution involves the values at the limits of the integration domain, in particular at the propellant surface $r=1$. Notice that due to (77), $J=0$ to the order $\epsilon$.

When the remaining terms in (79) are evaluated, taking into account that because $\sin \omega=0$,

we obtain

$$
\left[\hat{u}_{1}^{\prime}\right]_{x=1}=\left(\gamma A_{b_{0}}-\sigma-4+\frac{1}{r} \frac{\mathrm{d} F}{\mathrm{~d} r}\right) \frac{\cos \omega}{\gamma} \mathrm{e}^{\mathrm{i} \omega t+\sigma \tau},
$$

$$
G_{E}^{\prime}=\epsilon \pi\left(A_{b_{0}}-\sigma / \gamma\right) \cos \omega \mathrm{e}^{\mathrm{i} \omega t+\sigma \tau} .
$$

In the end section $E$ of the chamber we find rapid radial oscillations of vorticity and entropy associated with the time $\Sigma\left(r^{E}\right) / \epsilon$, defined in (18), required for a fluid particle to travel with the steady radial velocity from the propellant surface to the radius $r^{E}$. The additional residence time of the fluid particle in the nozzle, when it moves following very approximately the quasi-steady streamline, is $\epsilon^{\prime}$ times smaller 
than $\Sigma / \epsilon$; and thus, the viscous and heat conduction effects can be neglected in the nozzle, if terms of order $\epsilon$ are neglected. Then, in their motion between sections $E$ and $I$ along the steady streamlines the fluid particles will conserve the values of the entropy and, also, of the vorticity divided by $r$ that they had when they crossed the entrance section $E$. This is so because the pressure gradients are weak enough between $E$ and $I$ to allow us to neglect the baroclinic generation of vorticity.

The entropy $s^{\prime}$ and the vorticity $\omega^{\prime}$ are scaled with $c_{v}$ and $c_{b} / \epsilon a$, respectively. The wavelength of the entropy and vorticity waves in this region are of the same order, $\epsilon$, as in the chamber; but this is reduced at $I$ by a factor $r_{I} / r_{E}$ with the reduction of the cross-sectional area of the nozzle. Then, while the spatial entropy fluctuations at $I$ keep the order that they have at $E$, the velocity fluctuations are reduced by the factor $S_{I} / S_{E}$, because the vorticity and the wavelength are both reduced by the factor $r_{I} / r_{E}$.

In summary, in the low Mach number region between sections $E$ and $I$ the acoustic part of the velocity fluctuations is of order $\epsilon$, the spatial variations of the pressure are of order $\epsilon^{2}$, so that we can approximate $p^{\prime}$ by $\hat{p}_{E}^{\prime}$, and then $\hat{\rho}^{\prime}=\hat{\rho}_{E}^{\prime}=\hat{p}_{E}^{\prime} / \gamma$. The non-acoustic part $\bar{\rho}^{\prime}$ of $\rho^{\prime}$ is rapidly oscillating, with a wavelength of order $\epsilon$, so that its contribution to the first term in (73) is of order $\epsilon^{2}$. Then, (73) becomes

$$
\pi \epsilon^{\prime} \beta^{\prime} \frac{\mathrm{i} \omega}{\gamma} \hat{p}_{E}^{\prime}=G_{E}^{\prime}-G_{T}^{\prime}
$$

\subsection{Quasi-steady throat region of the nozzle}

In this region the residence time is small, of order $\epsilon t_{a}$, so that, as in the chamber, the contribution of the viscous stresses and the thermal conductivity are of order $\epsilon$, and thus, in first approximation, the entropy and the stagnation enthalpy are conserved along the streamlines, yielding

$$
\left(\frac{1+\lambda p_{I}^{\prime}}{\tilde{p}+\lambda p^{\prime}}\right)^{(\gamma-1) / \gamma}=\left(\frac{1+\lambda \rho_{I}^{\prime}}{\tilde{\rho}+\lambda \rho^{\prime}}\right)^{\gamma-1}=\frac{1+\lambda T_{I}^{\prime}}{\tilde{T}+\lambda T^{\prime}}=1+\frac{\gamma-1}{2} M^{2} .
$$

When writing (85) we have assumed that at station $I M^{2}=u^{2} / T$ is small compared with unity. The assumption of uniform pressure across the slowly varying crosssection in the nozzle region implies that $M$ is also uniform across the sections. The relations (85) should be linearized, around the mean values. If we split the oscillating variables into their acoustic and non-acoustic components, represented in this section with primes and an overbar, we obtain from the linearized form of (85)

$$
\left(\frac{1}{\tilde{p}}\right)^{(\gamma-1) / \gamma}=\left(\frac{1}{\tilde{\rho}}\right)^{\gamma-1}=\frac{1}{\tilde{T}}=1+\frac{\gamma-1}{2} \tilde{M}^{2},
$$

with $\tilde{M}=\tilde{u} / \sqrt{\tilde{T}}$, giving the mean flow variables in terms of $\tilde{p}(z)$.

For the acoustic oscillations, also uniform across the nozzle sections, we obtain

$$
\left(\hat{p}_{I}^{\prime}-\frac{\hat{p}^{\prime}}{\tilde{p}}\right)=\gamma\left(\hat{\rho}_{I}^{\prime}-\frac{\hat{\rho}^{\prime}}{\tilde{\rho}}\right)=\frac{\gamma}{\gamma-1}\left(\hat{T}_{I}^{\prime}-\frac{\hat{T}^{\prime}}{\tilde{T}}\right)=\gamma \tilde{M} \hat{M}^{\prime} /\left(1+\frac{\gamma-1}{2} \tilde{M}^{2}\right),
$$

with $\gamma \hat{\rho}_{I}^{\prime}=\gamma \hat{T}_{I}^{\prime} /(\gamma-1)=\hat{p}_{I}^{\prime}=\hat{p}_{E}^{\prime}=p_{0_{E}}$, and

$$
\hat{M}^{\prime}=\tilde{M}\left(\frac{\hat{u}^{\prime}}{\tilde{u}}-\frac{1}{2} \frac{\hat{T}^{\prime}}{\tilde{T}}\right) .
$$


The non-acoustic radial oscillations $\bar{\rho}^{\prime}, \bar{T}^{\prime}$ and $\bar{u}^{\prime}$ are given by the relations

$$
\bar{\rho}^{\prime} / \tilde{\rho}=\bar{\rho}_{I}^{\prime}, \quad \overline{2} \bar{u}^{\prime} / \tilde{u}=\bar{T}^{\prime} / \tilde{T}=\bar{T}_{I}^{\prime},
$$

based on $\bar{p}^{\prime}=0$, and therefore $\bar{M}^{\prime}=0$. These are valid through the whole quasi-steady region of the nozzle, and in particular at the throat. They allow us to calculate, in the throat region of the nozzle, the evolution along each streamline of the non-acoustic part of the fluctuations of the density, temperature and velocity in terms of the mean flow variables, given by (86) in terms of $\tilde{p}(z)$, and the fluctuations $\bar{\rho}_{I}^{\prime}$ and $\bar{T}_{I}^{\prime}$ at the station $I$ given by $\bar{T}_{I}^{\prime} / \tilde{T}_{I}=-\bar{\rho}_{I}^{\prime} / \tilde{\rho}_{I}^{\prime}=\bar{s}_{I}^{\prime} / \gamma$. Although the vorticity waves coming from the chamber are attenuated in the entrance region of the nozzle so that $\bar{u}_{I}^{\prime}=0$, new vorticity arises in the throat region due to the radial density oscillations coupled with the strong pressure gradient. This results in the values of $\bar{u}^{\prime}$ given by (89).

In order to calculate $p(z)=\tilde{p}(z)+\lambda \hat{p}^{\prime}(z)$, we shall use the mass conservation equation integrated across the section $z$ of the nozzle. This can be written as

$$
r_{b}^{2}(z)=2 \int_{0}^{\Psi_{b}} \frac{\mathrm{d} \Psi}{\rho u}
$$

where $\Psi_{b}$ is the constant value of $\Psi$ at the nozzle wall with radius $r_{b}(z)$, and $\rho u$ is evaluated in terms of $\Psi$ and $p$ using the relations (86)-(89).

At the nozzle throat $\mathrm{d} r_{b} / \mathrm{d} z=0$, which is equivalent to $\mathrm{d} r_{b} / \mathrm{d} p=0$ because $\mathrm{d} p / \mathrm{d} z \neq 0$. Therefore, the following relation is obtained (criticality condition at the nozzle throat):

$$
\int_{0}^{\Psi_{b}} \frac{\partial}{\partial p}\left(\frac{1}{\rho u}\right)_{\Psi} \mathrm{d} \Psi=\int_{0}^{\Psi_{b}}\left(p^{-\frac{3 \gamma+1}{2 \gamma}} \frac{1}{\gamma} \frac{1-M^{2}}{M^{3}}\right) \mathrm{d} \Psi=0 .
$$

The final form for the criticality condition at the throat section $T$ is

$$
\int_{0}^{\Psi_{b}} \frac{1-M^{2}}{M^{3}} \mathrm{~d} \Psi=0
$$

which, because $M$ is uniform across the section, leads to $M=1$; and this implies $\tilde{M}_{T}=1$ and $\hat{M}_{T}^{\prime}=0$.

Then, at the nozzle throat we obtain the well-known relations

$$
\tilde{p}_{T}^{(\gamma-1) / \gamma}=\tilde{\rho}_{T}^{\gamma-1}=\tilde{T}_{T}=\tilde{u}_{T}^{2}=2 /(\gamma+1)
$$

for the mean flow, and

$$
\hat{p}_{I}^{\prime}-\left(\frac{\hat{p}^{\prime}}{\tilde{p}}\right)_{T}=\hat{\rho}_{I}^{\prime}-\left(\frac{\hat{\rho}^{\prime}}{\tilde{\rho}}\right)_{T}=\hat{T}_{I}^{\prime}-\left(\frac{\hat{T}^{\prime}}{\tilde{T}}\right)_{T}=\left(\frac{\hat{u}^{\prime}}{\tilde{u}}\right)_{T}-\frac{\gamma-1}{2 \gamma}\left(\frac{\hat{p}^{\prime}}{\tilde{p}}\right)_{T}=0
$$

for the acoustic components; in these equations we can write $\gamma \hat{\rho}_{I}^{\prime}=\hat{T}_{I}^{\prime} \gamma /(\gamma-1)=$ $\hat{p}_{I}^{\prime}=\hat{p}_{E}^{\prime}=\cos \omega \mathrm{e}^{\mathrm{i} \omega t+\sigma \tau}$. The non-acoustic oscillations at the throat of the nozzle are given by (89), and thus they depend on $\Psi$ through the dependence of $\bar{\rho}_{I}^{\prime}$ and $\bar{T}_{I}^{\prime}$ on $\Psi$. (Their dependence on $r$ at the throat can be obtained from the definition $\Psi_{r}=r \rho u$.)

The mass flux $G_{T}^{\prime}$ can be evaluated now in terms of the profiles at the throat calculated above. Again, due to their rapid radial oscillations, the contributions of $\bar{\rho}^{\prime}$ and $\bar{u}^{\prime}$, associated with the entropy and vorticity waves, are of higher order than $\epsilon$ and can be neglected. Thus, we obtain

$$
G_{T}^{\prime}=\hat{G}_{T}^{\prime}=\epsilon 2 \pi\left(\frac{\hat{u}^{\prime}}{\tilde{u}}+\frac{\hat{\rho}^{\prime}}{\tilde{\rho}}\right)_{T}=\epsilon 2 \pi \frac{\gamma+1}{2 \gamma} \hat{p}_{E}^{\prime},
$$


where we have used (94), and $\hat{p}_{E}^{\prime}=p_{0_{E}}$. This can also be written as

$$
G_{T}^{\prime}=\hat{G}_{T}^{\prime}=\epsilon 2 \pi\left(A_{N_{0}}+1 / \gamma\right) \cos \omega \mathrm{e}^{\mathrm{i} \omega t+\sigma \tau},
$$

in terms of $A_{N_{0}}=(\gamma-1) / 2 \gamma$, the classical quasi-steady nozzle admittance function.

\section{Oscillation growth rate and frequency correction}

Now we have the elements necessary to determine the stability exponent $\sigma$, which includes the frequency correction to $\omega=n \pi$ given by $\sigma_{I}=\operatorname{Im}(\sigma)$, and the growth rate of the oscillations, $\sigma_{R}=\operatorname{Re}(\sigma)$. To do this, we use (83) and (96) in (84) and thus we obtain

$$
\sigma=\gamma\left(A_{b_{0}}-2 A_{N_{0}}\right)-2-\mathrm{i} \omega \beta,
$$

where we have defined $\beta=\beta^{\prime} \epsilon^{\prime} / \epsilon=\left(\Omega / \pi a^{3}\right) \epsilon^{\prime} / \epsilon$, of order unity. This expression for the stability exponent includes four contributions to the growth rate. The first term is due to the response of the combustion of the propellant under the action of the acoustic pressure oscillations. This effect can act as a driving mechanism of the combustion instability when $\operatorname{Re}\left(A_{b_{0}}\right)>0$. The second contribution, associated with acoustic energy losses through the nozzle, leads to damping, because $A_{N_{0}}=$ $(\gamma-1) / 2 \gamma>0$. The third contribution, -2 , is due to the mean flow/unsteady flow interaction; it leads to damping proportional to the gasification velocity coming from the propellant surface. This contribution can be better understood if the method of acoustic energy balance is used. Half of the damping, -1 , comes from the fact that the mean flow carries part of the acoustic energy through the nozzle. It is equal to the difference between the acoustic energy brought into the chamber by the mean flow and the energy sink corresponding to the nozzle exit. The other half, -1 , corresponds to what has been called flow turning by Culick (1975), and has also been included in our analysis. It results from the interaction with the mean flow of the unsteady flow field which must satisfy the no-slip condition. The flow turning is not related to the fluid viscosity and the result is independent of $v$; a discussion of the different ways to calculate the flow turning effects is presented in the Appendix. The last term in (97) is a frequency shift; it corresponds to an apparent elongation of the chamber to increase its volume by that of the entrance region of the nozzle.

An interesting result of the analysis is that none of the contributions to the growth rate, aside from the propellant admittance, $A_{b_{0}}$, depends, to the order $\epsilon$ considered, on the viscosity parameter $v$.

Although we were expecting an additional damping due to the energy losses through the nozzle, associated with the unsteady outflow produced by the vorticity and entropy waves, we have found that, due to their small wavelength, its effect is of higher order in our linear stability analysis.

\section{Discussion of the results and stability domain}

The analysis carried out in the previous sections gives a full description of the unsteady flow field. In figures 4 and 5 we show the transverse profiles of the fluctuating part of the axial velocity and the temperature, for the first and second modes evaluated for $\sigma=0$, at four different axial positions, for typical values of the parameters. Notice that near the chamber axis, once the vorticity and entropy waves are damped, we recover the pure acoustic solution. Numerical studies and experiments (see Avalon \& Comas 1991; Vuillot 1991; Vuillot \& Avalon 1991) predict the same behaviour with 

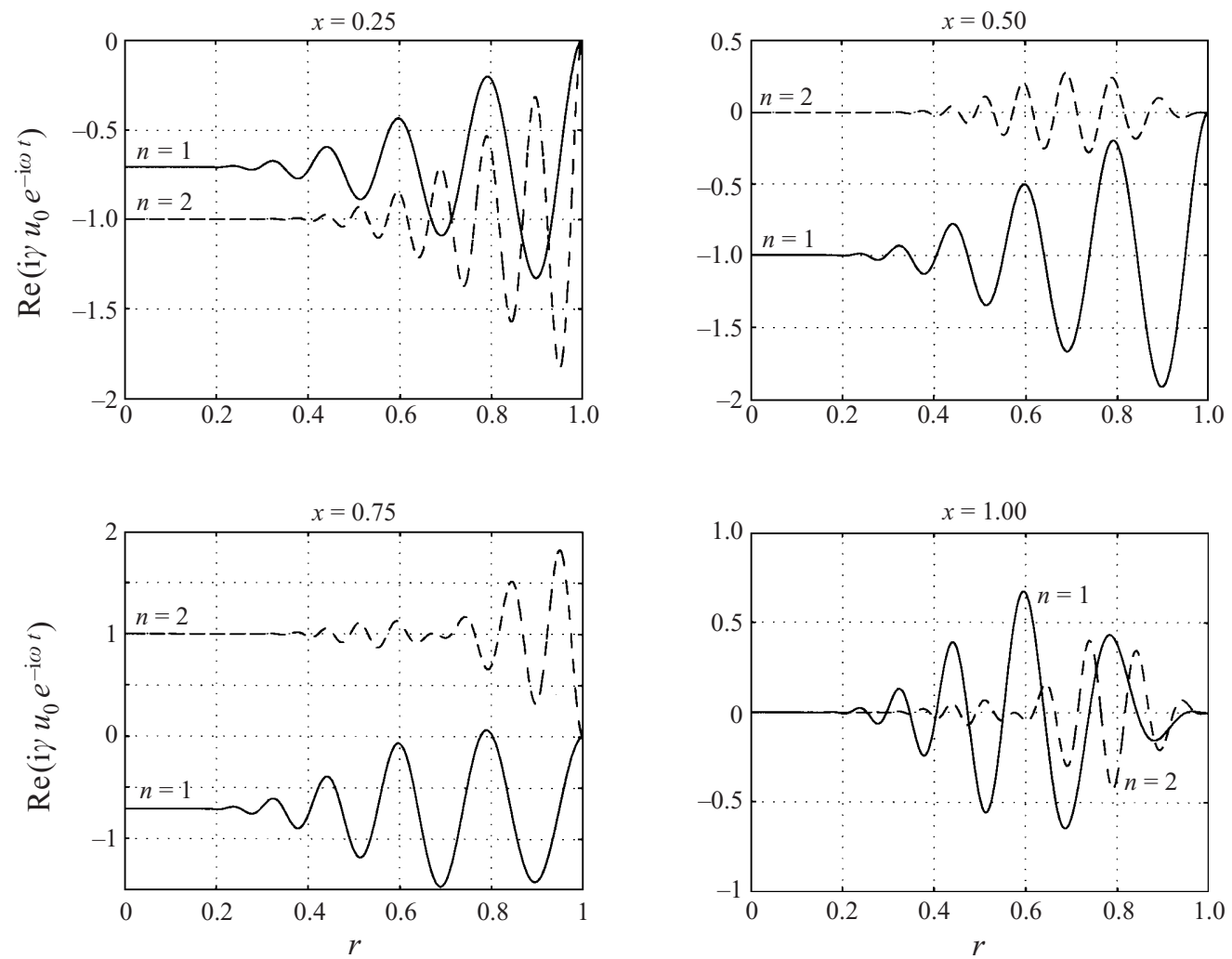

FIGURE 4. The zeroth-order axial velocity oscillation for the first (solid) and second (dashed) modes at four different axial positions with $\epsilon=0.1, v \pi^{2}=1$ and $\sigma=0$.

rapid radial oscillations near the chamber wall, recovering the classical acoustic plane wave solution near the chamber axis.

According to (97), the oscillations in the solid rocket motor become linearly unstable if $\operatorname{Re}\left(A_{b_{0}}\right)>(\gamma+1) / \gamma$. Here $\gamma$ is the ratio of the specific heats of the combustion products; a typical value of $\gamma$ is 1.3 used below in our calculations. The neutral stability boundary is given by

$$
\operatorname{Re}\left(A_{b_{0}}\right)=(\gamma+1) / \gamma
$$

in terms of the real part of the solid propellant admittance $A_{b_{0}}$, a function of the frequency of oscillation. This function needs to be obtained from experiments or computed using models of the solid propellant combustion subjected to gas-phase pressure oscillations.

For the longitudinal acoustic modes, the acoustic time $t_{a}=L / c_{b}$ is moderately large compared with the residence time, $t_{f}=l_{f} / V_{b}$, of the gas phase in the thin reaction layer of thickness $l_{f}=\alpha_{b} / V_{b}^{2}$. Therefore, a quasi-steady treatment of the gas phase appears justified. The analysis of the response function carried out by Denison \& Baum (1961) and, later, by Culick (1967) and Krier et al. (1968) used the assumption of quasi-steadiness in the gas-phase response. T'ien (1972), in his numerical analysis of the problem, retained unsteady effects in the gas phase, recovering the Denison $\&$ Baum model in the low-frequency range. The low-frequency value of $\operatorname{Re}\left(A_{b_{0}}\right)$ should correspond to the quasi-steady response of both the solid and gas phase; it 

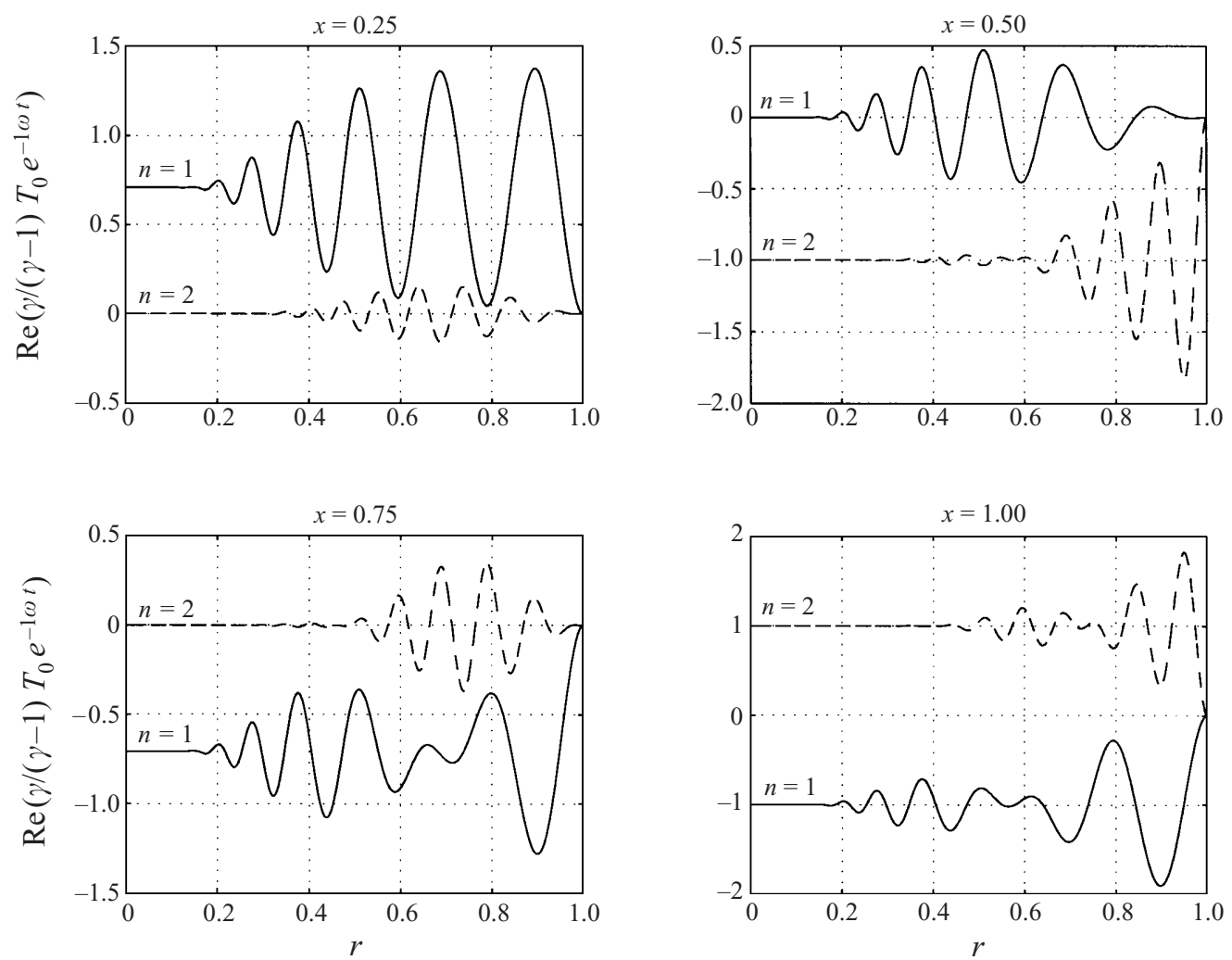

FIGURE 5. The zeroth-order temperature oscillation for the first (solid) and second (dashed) modes at four different axial positions with $\epsilon=0.1, v \pi^{2} / P r=1$ and $\sigma=0$.

is typically negative leading to damping oscillations. A more recent analysis, together with a discussion of the literature, is found in the paper by Clavin \& Lazimi (1992), where they use numerical and analytical techniques to describe, accounting for the effects of gas phase non-steadiness, the dependence of $A_{b_{0}}$ on frequency for the full frequency range. They showed how gas-phase unsteady effects substantially modify $A_{b_{0}}$, even at frequencies fairly small compared with $1 / t_{f}$.

As an example, we shall use, in our description of the solid propellant instabilities, the values of $A_{b_{0}}$ resulting from the Clavin \& Lazimi analysis. These values depend on a large number of parameters. In our calculations, leading to figures 6 and 7 , we have used for most of the parameters the values chosen by Clavin \& Lazimi in their calculations $\left(\gamma=1.3, \beta_{g}=8, L e=1, \beta_{g} / \beta_{s}=0.1\right.$ and $\left.\beta_{g} I=0.2\right)$, but we have varied two parameters, $r^{(s)}$ and $\beta_{g} \Delta \bar{\theta}_{s}$, that have a strong influence on $A_{b_{0}}$. One parameter, $r^{(s)}=\rho_{s}^{2} \alpha_{s} / \rho_{b}^{2} \alpha_{b}$, is the product of the square of the solid-to-gas density ratio and the ratio of the thermal diffusivities; $r^{(s)}$ is inversely proportional to the chamber pressure $p_{b}$, and typically ranges from 10 to 60 . The second parameter, $\beta_{g} \Delta \bar{\theta}_{s}$, is proportional to the non-dimensional activation energy of the gas-phase reaction and the difference in temperature between the initial solid temperature and its value in the solid-gas interface. The domain of instability increases with, and is sensitively dependent on, the value of this parameter. In our calculation we have considered the Lewis number $L e$ of the gas phase to be unity; the instability domain shown in figure 7 is enlarged if $L e>1$. 


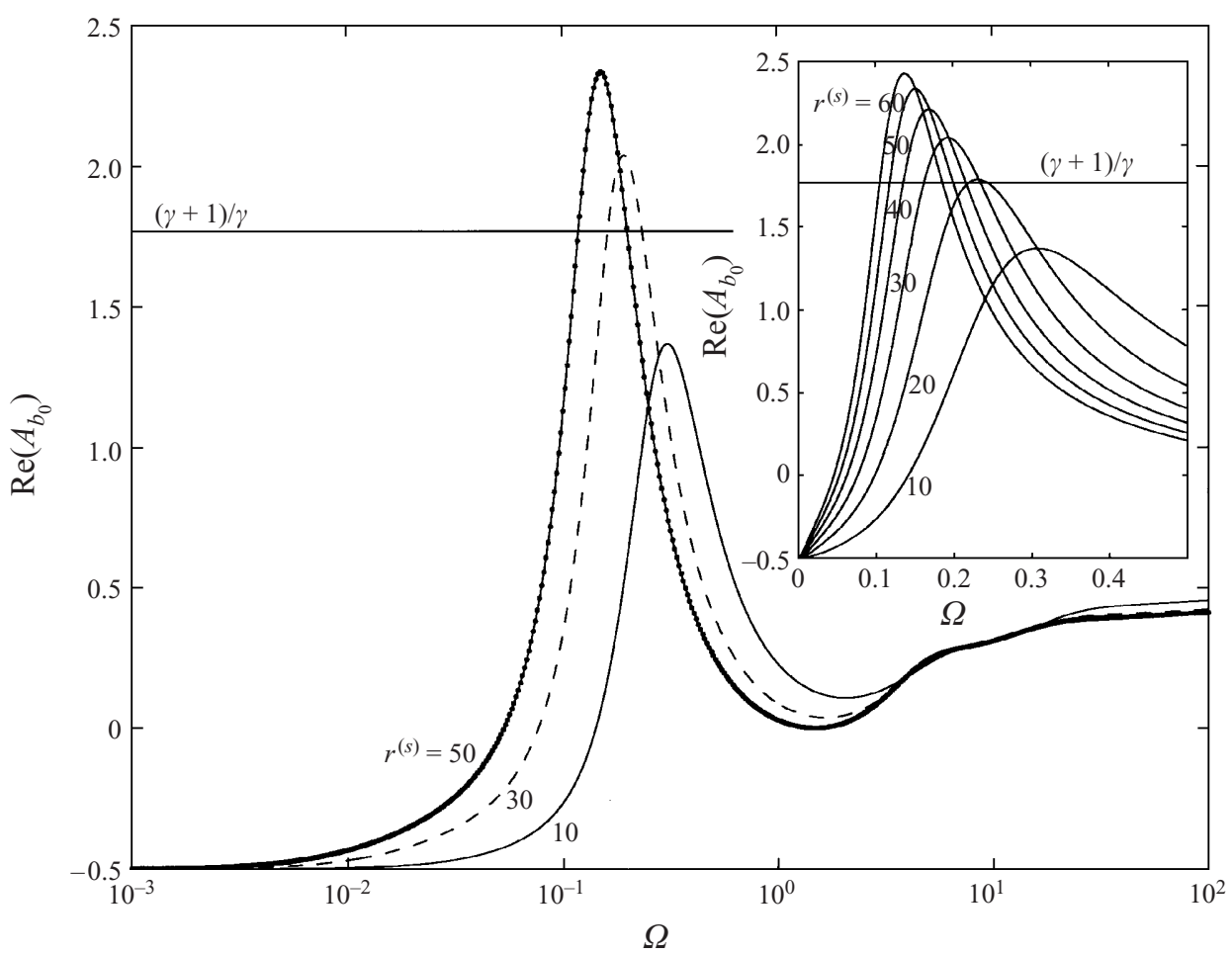

FIGURE 6. Real part of the solid propellant admittance function $A_{b_{0}}$ as a function of the angular frequency $\Omega=n \pi \epsilon v / P r$ for different solid-to-gas density ratios $r^{(s)}=\rho_{s}^{2} \alpha_{s} / \rho_{b}^{2} \alpha_{b}$ and $\beta_{g} \Delta \bar{\theta}_{s}=2.4$. The line $(\gamma+1) / \gamma$, with $\gamma=1.3$, defines the stability boundary. The inset is an enlargement for the frequency domain where $\operatorname{Re}\left(A_{b_{0}}\right)$ peaks.

The Clavin-Lazimi theory gives the results, plotted for various $r^{(s)}$ and $\beta_{g} \Delta \bar{\theta}_{s}=2.4$ in figure 6 , for the real part of the admittance as a function of their non-dimensional frequency $\Omega$, based on $t_{f}$. Notice that $\Omega$ is equal to our non-dimensional frequency $\omega$ times the ratio $t_{f} / t_{a}$. For the basic frequencies that we encounter in our analysis, $\omega=n \pi$, the resulting values of $\Omega$ are $n \pi t_{f} / t_{a}=n \pi \epsilon v / P r$. It turns out that the peak values of the real part of $A_{b_{0}}$ are found for values of $\Omega$ of order 0.2 , which correspond to not unreasonable values of $n \pi \epsilon v / P r$ for the first two modes, $n=1$ and 2 .

The stability boundary in the parameter plane $\left(r^{(s)}, \Omega\right)$ is given by $(98)$. It is plotted in figure 7, not only for the value $\beta_{g} \Delta \bar{\theta}_{s}=2.4$ used in figure 6 , but also for other values of $\beta_{g} \Delta \bar{\theta}_{s}$. Notice that $\Omega=n \pi\left(\alpha_{b} / V_{b}^{2}\right)\left(c_{b} / L\right)$ is pressure independent for the value $n_{g}=0.5$ used by Clavin \& Lazimi, and increases linearly with $n / L$.

The experimental results of Horton \& Price (1963), Traineau, Prevost \& Tarrin (1994) and Cauty (1999) show how the solid admittance function has a peak value, higher than $(\gamma+1) / \gamma \simeq 1.77$, in the frequency range of $100 \mathrm{~Hz}$ to $3000 \mathrm{~Hz}$, which belongs to the low-frequency domain corresponding to the longitudinal modes. Notice that if the classical result for $A_{b_{0}}=n_{g}-1<0$, as given by the linearization of the St. Robert's law, is used instability can never be achieved. But this result is only valid for very low frequencies, i.e. at most $10 \mathrm{~Hz}$, which are lower than the first longitudinal mode in solid propellant motors. The peak value of $\operatorname{Re}\left(A_{b_{0}}\right)$ is associated with growing unsteady effects, first in the solid phase and later in the gas phase. 


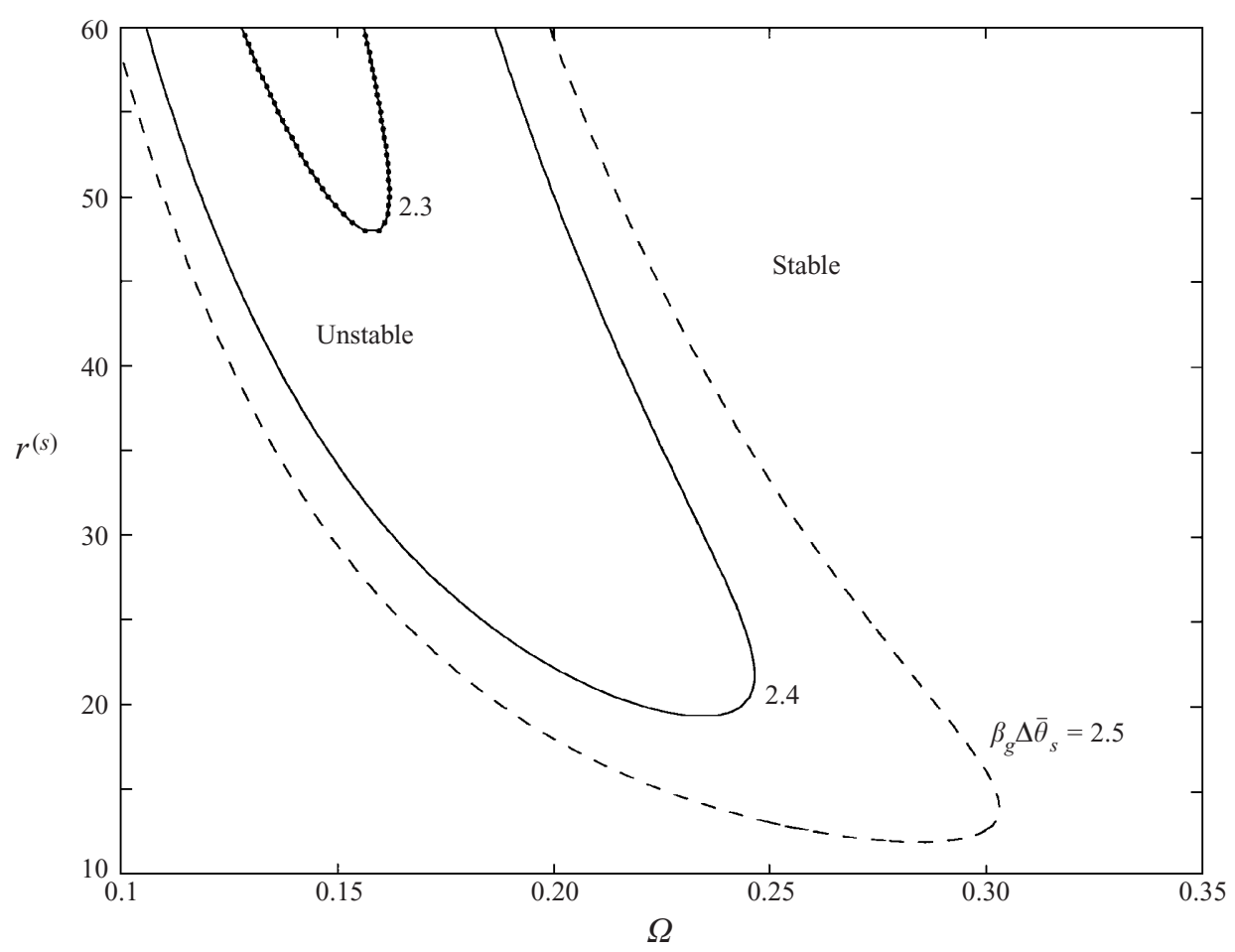

FIGURE 7. Stability boundary in the parameter plane $\left(r^{(s)}, \Omega\right)$ for various values of $\beta_{g} \Delta \bar{\theta}_{s}$. The solid line corresponds to the value, $\beta_{g} \Delta \bar{\theta}_{s}=2.4$, used in the determination of the response function given in figure 6.

\section{Conclusions}

We have carried out a linear analysis of the evolution of the longitudinal acoustic oscillations within the chamber of solid propellant rockets with an axis-symmetric configuration. The analysis is based on the assumption that the chamber is slender $\left(\epsilon^{\prime}=a / L \ll 1\right)$ and axially symmetric, and that the Mach number, $M_{b}$, of the burned gases, when they leave the thin reaction layer radially, is small enough that the Mach number of the axial steady flow $\epsilon=M_{b} / \epsilon^{\prime}$, typically of order $\epsilon^{\prime}$, is also small compared with 1 . Then, as stated in the Introduction, within the chamber we encounter vorticity and entropy waves, involving several time and length scales, thus enabling us to use the method of multiple scales to obtain an analytical description of the zeroth-order unsteady flow field, and the linear growth rate of the disturbances. The method can be used in a straightforward way to obtain higher-order terms in the perturbation scheme.

The origin of the vorticity waves that we describe in our analysis was clarified by Flandro, beginning with his work of (1989), where the radial oscillations are described assuming the radial velocity to be constant. In his more recent review paper of (1995), he allows for the radial variations of $V$ to calculate the vorticity waves, and predicts a value of $\sigma$ that differs from our result given in (97), mainly due to an error in his handling of the boundary condition for $v^{\prime}$ at the propellant surface.

The presence of the vorticity and entropy waves, with a wavelength $l_{0}=\epsilon a$, independent of viscosity and heat conduction, but an amplitude that decreases at fixed $r$ with increasing values of $v$, leads to a growth rate $\sigma_{R}$ of the acoustic disturbances 
that turns out to be independent of $v$. This is due to the fact that the effects of these waves on the nozzle admittance cancel in a first approximation, as a result of their rapid radial variation around the mean. To obtain the growth rate of the disturbances we have used the boundary conditions, on the wall and at the exit of the chamber, for the differential equations that describe the time and space evolution of the disturbances. It could also be derived, as indicated in the Appendix, by a global acoustic balance method involving surface integrals. This procedure was used by Mazon (1996), where the effects of particles on the damping was also taken into account.

It is important to observe that in our analysis the only term which can trigger instabilities is the response of the solid propellant burning. We have found that for typical values of the parameters of solid propellant rockets, the longitudinal modes can be unstable. More accurate combustion models of the solid propellant response to pressure oscillations will lead to more exact admittance functions, and better linear instability predictions.

We have considered in our analysis that the outer toroidal recirculating region in the entrance of the nozzle, shown by numerical descriptions of the basic flow and sketched in figure 3 , does not periodically shed gases to modify significantly the nozzle admittance.

The linear stability analysis presented here is the basis for the nonlinear analysis by Perrin $\&$ Clavin as presented in Perrin (1996). Note that when $\lambda$ grows to $O(\epsilon)$ nonlinear effects will modify the evolution equations (54) and (55) for the amplitude, $\bar{u}_{0}$ and $\bar{T}_{0}$, of the radial oscillations of velocity and temperature in the bulk of the chamber. In addition, the nonlinear effects could also introduce changes in $G_{E}^{\prime}$ due to the term $\lambda \int \bar{\rho}^{\prime} \bar{u}^{\prime} \mathrm{d} S_{E}$; the combined action of the vorticity and entropy waves will lead in the nonlinear theory to coupling of the acoustic modes.

This work was supported by the Spanish CICYT, under Contract No. PB94-0400, and by INTA under the Project Mezcla y Combustión.

\section{Appendix. Flow turning}

We shall show, in this Appendix, the relation between our analysis and the method of evaluation by Culick of the flow-turning effects on the acoustic instabilities. After the introduction by Culick (1975) of this idea much research has been carried out to try to clarify this phenomenon; see Baum \& Levine (1986), Kuentzmann (1991), Van Moorhem (1982) and Vuillot \& Avalon (1991). The technique of Culick (1970, 1973, 1975) and Culick \& Yang (1992), which is based on the integral form of the equations, is useful for the physical understanding of the stability mechanisms. It includes a strong over-simplification: the assumption of irrotational acoustics to describe the unsteady field. If rotational terms are kept, the formula derived by Culick to compute the stability exponent takes the following form (rewritten in our notation):

$$
\begin{aligned}
& -\epsilon 2 \mathrm{i} \omega \sigma \int_{0}^{1} \int_{0}^{1} \psi_{n} p^{\prime} r \mathrm{~d} r \mathrm{~d} x=\epsilon \mathrm{i} \omega \int_{0}^{1}\left[\psi_{n} p^{\prime} \tilde{V}+\gamma \psi_{n} v^{\prime}\right]_{r=1} \mathrm{~d} x \\
& +\mathrm{i} \omega \int_{0}^{1}\left[\epsilon \psi_{n} p^{\prime} \tilde{U}+\gamma \psi_{n} u^{\prime}\right]_{x=1} r \mathrm{~d} r+\epsilon \gamma \int_{0}^{1} \int_{0}^{1} \tilde{V} \frac{\partial u^{\prime}}{\partial r} \frac{\mathrm{d} \psi_{n}}{\mathrm{~d} x} r \mathrm{~d} r \mathrm{~d} x+O\left(\epsilon^{2}\right),
\end{aligned}
$$

where $p^{\prime}=\psi_{n} \mathrm{e}^{\mathrm{i} \omega t+\sigma \tau}$ and $\psi_{n}=\cos \left(\omega_{n} x\right)$, with $\omega=\omega_{n}=n \pi$, are the longitudinal acoustic modes for the closed cylindrical chamber. The last integral is due to the 
rotational flow field and must be evaluated, like the other integrals in (A 1), using our description of $u^{\prime}$, given in (47), together with the boundary condition (16) and the relation between the classical acoustic velocity oscillation at the chamber exit and the pressure oscillation, $\hat{u}^{\prime} / \tilde{U}=\epsilon A_{N_{E}} \hat{p}^{\prime}=\epsilon\left(A_{N_{0}}+\mathrm{i} \omega \beta / 2 \gamma\right) \hat{p}^{\prime}$. Thus, we obtain $\sigma=\gamma\left(A_{b}-2 A_{N_{E}}\right)-2$, the value for the stability exponent given in (97).

Culick (1973) also developed an approximate one-dimensional theory that surprisingly leads to the same result, due to the presence of a term named the one-dimensional flow turning correction. Flandro (1995) has already given evidence for the justification of this correction in terms of the contributions of the two-dimensional rotational flow. The flow turning is, as pointed out by Flandro, a volumetric effect represented by the last term in (A 1). We shall briefly show here how, beginning with the threedimensional description (A 1), we can reduce the volume integral in (A 1) to a contour integral identical to the one in Culick's one-dimensional flow formulation. Of the various contributions to the last integral in (A 1), associated with rotational flow field effects, if we leave out terms of higher order, the main contribution comes from the term

$$
\int_{V} \tilde{V} \frac{\partial u^{\prime}}{\partial r} \frac{\mathrm{d} \psi_{n}}{\mathrm{~d} x} \mathrm{~d}\left(\pi r^{2}\right) \mathrm{d} x=\int_{V} \tilde{V} \xi_{r} \frac{\partial u^{\prime}}{\partial \xi} \frac{\mathrm{d} \psi_{n}}{\mathrm{~d} x} \mathrm{~d}\left(\pi r^{2}\right) \mathrm{d} x=K \mathrm{e}^{\mathrm{i} \omega t+\sigma \tau},
$$

where, if we take into account that $\partial u^{\prime} / \partial \xi=-\mathrm{i} \omega \bar{u} \mathrm{e}^{\mathrm{i} \omega(t-\xi)+\sigma \tau}$, and remembering the definition (18) of the rapid transverse variable $\xi$,

$$
K=\int_{0}^{1}\left[\int_{0}^{1}-\frac{1}{\epsilon} \mathrm{i} \omega \bar{u} \frac{\mathrm{d} \psi_{n}}{\mathrm{~d} x} \mathrm{e}^{-i \omega \xi} 2 \pi r \mathrm{~d} r\right] \mathrm{d} x .
$$

Using again integration by parts for $\epsilon \rightarrow 0$, we obtain

$$
K=\int_{0}^{1}-\frac{1}{\epsilon} \mathrm{i} \omega\left(\frac{\mathrm{d} \psi_{n}}{\mathrm{~d} x}\right)\left[-\frac{1}{\mathrm{i} \omega \xi_{r}} \bar{u} \mathrm{e}^{-\mathrm{i} \omega \xi} 2 \pi r\right]_{r=0}^{r=1} \mathrm{~d} x .
$$

The value of the integrand is obviously zero at $r=0$, and only the contribution at the chamber wall remains. If we recall the no-slip boundary condition (32) at the propellant surface, we conclude that there the amplitude of the vorticity wave must be equal to the amplitude of the acoustic wave, namely $\left(\bar{u} \mathrm{e}^{-\mathrm{i} \omega \xi}\right)_{r=1}=-(\mathrm{i} / \gamma \omega)\left(\mathrm{d} \psi_{n} / \mathrm{d} x\right)$. Then,

$$
K=\int_{0}^{1}\left(\frac{\mathrm{d} \psi_{n}}{\mathrm{~d} x}\right)\left[\frac{1}{\epsilon \xi_{r}} \frac{\mathrm{i}}{\gamma \omega}\left(\frac{\mathrm{d} \psi_{n}}{\mathrm{~d} x}\right) 2 \pi\right]_{r=1} \mathrm{~d} x=\int_{0}^{1} \frac{\mathrm{i}}{\gamma \omega}\left(\frac{\mathrm{d} \psi_{n}}{\mathrm{~d} x}\right)^{2}[-2 \pi \tilde{V}(1)] \mathrm{d} x,
$$

where the factor $[-2 \pi \tilde{V}(1)]$ in (A 5) represents the volumetric flux entering the chamber per unit length and time. This expression for $K$ is identical to the flow turning term in Culick's one-dimensional analysis. Thus, we conclude that the threedimensional, global balance, formulation is accurate if the rotational effects are retained, and coincides with the results given by the analysis performed with the differential equations and with the one-dimensional acoustic balance method. The details of the vortical oscillating field need not be known to calculate its contribution. We need only to know its structure and the fact, used in the evaluation of $K$, that the wavelength of the radial oscillation is small, of order $\epsilon$, compared with the radius of the chamber. This integral analysis also shows how the results are independent of the value of the viscosity. The flow turning is due to the no-slip condition and to the interaction of the mean flow and the acoustic flow field. 


\section{REFERENCES}

Avalon, G. \& Comas, P. 1991 Simulative study of the unsteady flow inside a solid rocket motor. AIAA/SAE/ASME/ASEE 27th Joint Propulsion Conference and Exhibit, Sacramento, CA (USA), June 24-27, 1991.

Balakrishnan, G., Liñán, A. \& Williams, F. A. 1992 Rotational inviscid flow in laterally burning solid-propellant rocket motors. J. Propulsion Power 8, 1167-1176.

Baum, J. D. \& Levine, J. N. 1986 Numerical study of flow-turning phenomenon. AIAA Paper 86-0533.

Bender, C. M. \& Orszag, S. A. 1978 Advanced Mathematical Methods for Scientists and Engineers. McGraw-Hill.

CAUTY, F. 1999 Solid-propellant combustion response function from direct measurement methods: ONERA experience. J. Combust. Power 15, 837-843.

Clavin, P. \& Lazimi, D. 1992 Theoretical analysis of oscillatory burning of homogeneous solid propellant including non-steady gas phase effects. Combust. Sci. Tech. 83, 1-32.

Crocco, L. \& Cheng, S. I. 1956 Supercritical gaseous discharge with high frequency oscillations. In Theory of Combustion Instability in Liquid Propellant Rocket Motors. AGARDograph 8, Appendix B.

Crocco, L. \& Sirignano, W. A. 1967 Behaviour of Supercritical Nozzles under Three-Dimensional Oscillatory Condition. AGARDograph 117, Princeton University, Princeton, New Jersey.

Culick, F. E. 1966 Rotational axisymmetric mean flow and damping of acoustic waves in solid propellant rocket motors. AIAA J. 4, 1462-1464.

Culick, F. E. C. 1967 Calculation of the admittance function for a burning surface. Astronautica Acta 13, 221-237.

Culick, F. E. 1970 The stability of longitudinal oscillations with pressure and velocity coupling in solid propellant rocket motors. Combust. Sci. Tech. 2, 179-201.

Culick, F. E. 1973 The stability of one-dimensional motions in a rocket motor. Combust. Sci. Tech. 7, $165-175$.

Culick, F. E. 1975 Stability of three-dimensional motions in a combustion chamber. Combust. Sci. Tech. 10, 109-124.

Culick, F. E. \& YANG, V. 1992 Prediction of stability of unsteady motions in solid propellant rocket motors. Prog. Astronaut. Aeronaut. 143, 719-779.

Denison, M. R. \& BAuM, E. 1961 A simplified model of unstable burning in solid propellants. ARS J. 31, 1112-1122.

Dunlap, R., Willoughby, P. G. \& Hermsen, R. W. 1974 Flowfield in the combustion chamber of a solid propellant rocket motor. AIAA J. 12, 1440-1441.

Flandro, G. A. 1974 Solid propellant acoustic admittance corrections. J. Sound Vib. 36, 297-312.

Flandro, G. A. 1989 Effects of vorticity transport on axial acoustic waves in a solid propellant rocket chamber. ASME Winter Meeting, San Francisco, California, December 1989.

Flandro, G. A. 1995 Effects of vorticity on rocket combustion stability. J. Propulsion Power 11, 607-625.

Horton, M. D. \& Price, E. W. 1963 Combustion instability. In Ninth Symp. (Intl) on Combustion, pp. 303-310. The Combustion Institute.

Krier, H., T'ien, J. S., Sirignano, W. A. \& Summerfield, M. 1968 Nonsteady burning phenomena of solid propellants: theory and experiments. AIAA J. 6, 278-285.

Kuentzmann, P. 1991 Combustion Instabilities. AGARD Lecture Series No. 180.

Lupoglazoff, N. \& Vuillot, F. 1991 Two-dimensional numerical simulation of the stability of a solid propellant rocket motor. AIAA 29th Aerospace Science Meeting, Reno, NE (USA), January 7-10, 1991.

Majdalani, J. \& Van Moorhem, W. K. 1997a Multiple scale solution to the acoustic boundary layer in solid rocket motors. J. Propulsion Power 13, 186-193.

Majdalani, J. \& Van Moorhem, W. K. $1997 b$ An improved time-dependent flow field solution for solid rocket motors. AIAA Paper 97-27117.

Mazon, D. 1996 Etude de la stabilit linaire du mode acoustique longitudinal. Doctoral Thesis, Universit de Provence (Aix-Marseille I).

Perrin, J. L. 1996 Etude non linaire des nstabilits acoustiques longitudinales dans les moteurs propergol solide. Doctoral Thesis, Universit de Provence (Aix-Marseille I). 
TAYLOR, G. I. 1956 Fluid flow in regions bounded by porous surfaces. Proc. R. Soc. Lond. A 234, 456-475.

T'IEN, J. S. 1972 Oscillatory burning of solid propellants including gas phase time lag. Combust. Sci. Tech. 5, 47-54.

T'IEN, J. S. 1984 Theoretical analysis of combustion instability. In Fundamentals of Solid-Propellant Combustion. Progress in Astronautics and Aeronautics, Vol. 90, Chap. 14, pp. 791-840.

Traineau, J. C., Prevost, M. \& Tarrin, P. 1994 Experimental low and medium frequency determination of solid propellants pressure-coupled response function. AIAA Paper 94-3043.

VAN MOORHEM, W. K. 1982 Flow-turning in solid-propellant rocket combustion stability analysis. AIAA J. 20, 1420-1425.

Vuillot, F. 1991 Numerical computation of acoustic boundary layers in large solid propellant Space Booster. AIAA Paper 91-0206.

Vuillot, F. \& Avalon, G. 1991 Acoustic boundary layers in solid propellant rocket motors using Navier-Stokes equations. J. Propulsion Power 7, 231-239.

Vuillot, F. \& Kuentzmann, P. 1986 Flow turning and admittance correction: an experimental comparison. J. Propulsion Power 2, 345-353.

ZhaO, Q., StaAb, P. L., Kassoy, D. R. \& KirKKopru, K. 2000 Acoustically generated vorticity in an internal flow. J. Fluid Mech. 413, 247-285. 